\title{
Modeling particle size distribution in emulsion polymerization reactors
}

\author{
H. M. Vale and T. F. McKenna \\ CNRS-LCPP/ESCPE-Lyon, BP 2077, 43 Blvd du 11 novembre 1918, 69616 \\ Villeurbanne Cedex, France
}

\begin{abstract}
A review of the use and limitations of Population Balance Equations (PBE) in the modeling of emulsion polymerisation (EP), and in particular of the particle size distribution of the dispersed system is presented. After looking at the construction of the general form of PBEs for EP, a discussion of the different approaches used to model polymerization kinetics is presented. Following this, specific applications are presented in terms of developing a two-dimensional PBE for the modeling of more complex situations (for example the particle size distribution, PSD, and the composition of polymerizing particles). This review demonstrates that while the PBE approach to modeling EP is potentially very useful, certain problems remain to be solved, notably: the need to make simplifying assumptions about the distribution of free radicals in the particles in order to limit the computation complexity of the models; and the reliance of full models on approximate coagulation models. The review finishes by considering the different numerical techniques used to solve PBEs.
\end{abstract}

\section{Keywords}

Particle size distribution; Population balance; Coagulation; Emulsion polymerization; Modeling 


\section{Table of Contents}

Nomenclature

1. Introduction

2. Formulation of the population balance

2.1 Principles of PBEs

2.2 Kinetics

2.2.1 Introduction

2.2.2 Zero-one model

2.2.3 Pseudo-bulk model

2.2.4 Average number of radicals per particle

2.3 Multicomponent systems

2.3.1 Pseudo-homopolymerization approach

2.3.2 Multicomponent approach

2.4 Other types of reactors

2.4.1 Tubular reactors

2.4.2 Non-ideal stirred tank reactors

3. Coagulation modeling

3.1 Coagulation mechanisms

3.2 Modeling coagulation rate coefficients

3.2.1 DLVO-based models

3.2.1.1 Overview of the DLVO approach

3.2.1.2 Limitations of the DLVO approach

3.2.1.3 Experimental validation

3.2.2 Non DLVO-based models

4. Numerical solution of the population balance model

4.1 Introduction

4.2 Finite elements

4.3 Finite differences/volumes

5. Conclusions

Acknowledgments

References 


\section{Nomenclature}

a dimensionless entry frequency

c pseudo-first-order rate coefficient for termination $\left(\mathrm{s}^{-1}\right)$

$c \quad$ number of monomers

$C_{i} \quad i$ th cell of the particle size domain

$D \quad$ axial dispersion coefficient $\left(\mathrm{m}^{2} \mathrm{~s}^{-1}\right)$

$f_{n}(v, t) \quad$ number density function for particles having $n$ radicals (part $\mathrm{m}^{-3} \mathrm{~m}^{-3}$ )

$F(x, \mathbf{r}, t) \quad$ number density function $\left(\operatorname{part~}^{-3}[x]^{-1}\right)$

$F_{\mathbf{m}}(\mathbf{m}, t) \quad$ number density function (part $\mathrm{m}^{-3} \mathrm{~kg}^{-c}$ )

$F_{\mathbf{w}}(m, \mathbf{w}, t) \quad$ number density function (part $\mathrm{m}^{-3} \mathrm{~kg}^{-1}$ )

$\bar{F}_{i}(t) \quad$ average value of $F(r, t)$ over $C_{i}\left(\operatorname{part~}^{-3} \mathrm{~m}^{-1}\right)$

$G \quad$ hydrodynamic interaction function

I $\quad$ ionic strength $\left(\mathrm{mol} \mathrm{m}^{-3}\right)$

$j_{\text {crit }} \quad$ critical degree of polymerization for particle formation by homogeneous nucleation

$k_{\mathrm{B}} \quad$ Boltzmann's constant $\left(\mathrm{J} \mathrm{K}^{-1}\right)$

$k_{\text {des }} \quad$ desorption frequency $\left(\mathrm{s}^{-1}\right)$

$k_{\mathrm{dM}} \quad$ rate coefficient for desorption of monomeric radicals from particles $\left(\mathrm{s}^{-1}\right)$

$k_{\mathrm{fM}} \quad$ rate coefficient for transfer to monomer $\left(\mathrm{m}^{3} \mathrm{~mol}^{-1} \mathrm{~s}^{-1}\right)$

$k_{\mathrm{p}} \quad$ propagation rate coefficient $\left(\mathrm{m}^{3} \mathrm{~mol}^{-1} \mathrm{~s}^{-1}\right)$

$k_{\mathrm{p} i j} \quad$ propagation rate coefficient for radical ending in monomer $i$ adding monomer $j\left(\mathrm{~m}^{3} \mathrm{~mol}^{-1} \mathrm{~s}^{-1}\right)$

$k_{\mathrm{t}} \quad$ termination rate coefficient $\left(\mathrm{m}^{3} \mathrm{~mol}^{-1} \mathrm{~s}^{-1}\right)$

$k_{\mathrm{t}}^{i j} \quad$ termination rate coefficient between chains of length $i$ and $j\left(\mathrm{~m}^{3} \mathrm{~mol}^{-1} \mathrm{~s}^{-1}\right)$

$K \quad$ rate coefficient of volume growth $\left(\mathrm{m}^{3} \mathrm{~s}^{-1}\right)$

$L \quad$ length of the reactor (m)

$m \quad$ dimensionless desorption frequency

$m \quad$ total mass of polymer in a particle $(\mathrm{kg})$

$m_{i} \quad$ mass of polymer $i$ in a particle $(\mathrm{kg})$

m vector of polymer masses $(\mathrm{kg})$

$[\mathrm{M}]_{\mathrm{p}} \quad$ concentration of monomer in a particle $\left(\mathrm{mol} \mathrm{m}^{-3}\right)$ 


\begin{tabular}{|c|c|}
\hline$M$ & number of finite volumes \\
\hline$M W$ & molecular weight $\left(\mathrm{kg} \mathrm{mol}^{-1}\right)$ \\
\hline$n$ & number of radicals per particle \\
\hline $\bar{n}$ & average number of radicals per particle \\
\hline$N_{0}$ & initial number of particles (part) \\
\hline$N_{\mathrm{A}}$ & Avogradro's number $\left(\mathrm{mol}^{-1}\right)$ \\
\hline$N_{i}$ & number of particles in $C_{i}\left(\right.$ part $\left.\mathrm{m}^{-3}\right)$ \\
\hline$p_{i}$ & probability of a particle having a radical ending in monomer $i$ \\
\hline$Q$ & volumetric flow rate $\left(\mathrm{m}^{3} \mathrm{~s}^{-1}\right)$ \\
\hline$r$ & unswollen radius of a particle $(\mathrm{m})$ \\
\hline$r_{\mathrm{co}}$ & cross-over radius $(\mathrm{m})$ \\
\hline$r_{i}$ & average unswollen radius of $C_{i}(\mathrm{~m})$ \\
\hline$r_{\max }$ & upper bound of the unswollen radius domain (m) \\
\hline$r_{\mathrm{s}}$ & swollen radius of a particle $(\mathrm{m})$ \\
\hline$\Delta r_{i}$ & size of $C_{i}(\mathrm{~m})$ \\
\hline $\mathbf{r}$ & position coordinate $(\mathrm{m})$ \\
\hline$R$ & center-to-center distance between particles (m) \\
\hline$R_{i}$ & average number of growing chains of length $i$ per particle \\
\hline$R_{\text {nuc }}$ & rate of particle nucleation (part $\mathrm{m}^{-3} \mathrm{~s}^{-1}$ ) \\
\hline ई & rate of change of the external coordinates $\left(\mathrm{m} \mathrm{s}^{-1}\right)$ \\
\hline $\mathfrak{R}$ & net rate of particle generation (part s $\mathrm{m}^{-1}[x]^{-1}$ ) \\
\hline $\mathfrak{R}_{\text {coag }}^{-}$ & particle depletion rate due to coagulation (part s $\mathrm{s}^{-1} \mathrm{~m}^{-3}[x]^{-1}$ ) \\
\hline $\mathfrak{R}_{\text {coag }}^{+}$ & particle formation rate due to coagulation (part s ${ }^{-1} \mathrm{~m}^{-3}[x]^{-1}$ ) \\
\hline$t$ & time $(s)$ \\
\hline$T$ & temperature $(\mathrm{K})$ \\
\hline$u(\mathbf{r})$ & spatial distribution of the particles at $t=0\left(\mathrm{~m}^{-3}\right)$ \\
\hline$u_{z}$ & average axial velocity $\left(\mathrm{m} \mathrm{s}^{-1}\right)$ \\
\hline$v$ & unswollen volume of a particle $\left(\mathrm{m}^{3}\right)$ \\
\hline$v_{i}$ & unswollen volume corresponding to $r_{i}\left(\mathrm{~m}^{3}\right)$ \\
\hline$v_{\mathrm{s}}$ & swollen volume of a particle $\left(\mathrm{m}^{3}\right)$ \\
\hline$V$ & volume $\left(\mathrm{m}^{3}\right)$ \\
\hline$V_{\mathrm{R}}$ & electrostatic repulsion energy $(\mathrm{J})$ \\
\hline
\end{tabular}




$\begin{array}{ll}V_{\mathrm{T}} & \text { total particle interaction energy }(\mathrm{J}) \\ w(x) & \text { size distribution of the particles at } t=0\left([x]^{-1}\right) \\ w_{i} & \text { mass fraction of polymer } i \text { in a particle } \\ \mathbf{w} & \text { vector of mass fractions } \\ W & \text { Fuchs' stability ratio } \\ x & \text { size coordinate }([x]) \\ z & \text { critical degree of polymerization for entry into particles or micelles } \\ z & \text { axial coordinate }(\mathrm{m})\end{array}$

\section{Subscripts and superscripts}

0

surface

d diffuse layer

in reactor inlet

m monomer

M monomeric radical

nuc nucleated particles

out reactor outlet

$\mathrm{P} \quad$ polymeric radical

w aqueous phase

\section{Greek Symbols}

$\alpha$

dimensionless entry frequency

$\beta$

coagulation rate coefficient $\left(\mathrm{m}^{3} \operatorname{part}^{-1} \mathrm{~s}^{-1}\right)$

$\delta$

Dirac delta-function

$\delta_{i, j}$

Kronecker delta

$\varepsilon$

rate of energy dissipation $\left(\mathrm{m}^{2} \mathrm{~s}^{-3}\right)$

$\phi_{\mathrm{pp}}$

volume fraction of polymer in a particle

$\phi_{\mathrm{w}} \quad$ volume fraction of the aqueous phase

$\Phi \quad$ particle flux (part $\left.\mathrm{m}^{-3} \mathrm{~s}^{-1}\right)$

$\eta \quad$ aggregation function, Eq. (38)

$\kappa \quad$ inverse double layer thickness $\left(\mathrm{m}^{-1}\right)$

$\mu \quad$ viscosity (Pa s) 


$\begin{array}{ll}\rho & \text { total entry frequency of radicals into particles }\left(\mathrm{s}^{-1}\right) \\ \rho_{\mathrm{E}} & \text { entry frequency of monomeric radicals into particles }\left(\mathrm{s}^{-1}\right) \\ \rho_{\mathrm{I}} & \text { entry frequency of initiator-derived radicals into particles }\left(\mathrm{s}^{-1}\right) \\ \rho_{\mathrm{p}} & \text { density of the polymer }\left(\mathrm{kg} \mathrm{m}^{-3}\right) \\ \sigma & \text { surface charge density }\left(\mathrm{C} \mathrm{m}^{-2}\right) \\ \Omega & \text { coordinate domain } \\ \psi & \text { electric potential }(\mathrm{V})\end{array}$

\section{Abbreviations}

B

first order backward scheme

BC boundary condition

C2 second order central scheme

CFD computational fluid dynamics

CLD chain-length-dependent

CMC critical micellar concentration

CSTR continuous stirred tank reactor

DAE differential algebraic equation

DLVO Deryaguin-Landau-Verwey-Overbeek (theory)

DPB discretized population balance

EP emulsion polymerization

FD finite difference

FE finite element

FV finite volume

HHF Hogg-Healy-Fürstenau (theory)

HSC high solid content

IC initial condition

MWD molecular weight distribution

OCFE orthogonal collocation on finite elements

ODE ordinary differential equation

PB pseudo-bulk

PBE population balance equation

PBM population balance model

PDE partial differential equation 
PSD particle size distribution

RTD residence time distribution

STR stirred tank reactor

WENO weighted essentially non-oscillatory 


\section{Introduction}

First implemented at an industrial scale during World War II as a means to overcome the urgent need for synthetic rubber, emulsion polymerization developed rapidly and is nowadays the process of choice to prepare millions of tonnes of synthetic polymer latexes. Most synthetic elastomers and water-borne coatings, and a significant part of plastics, are, in fact, prepared by this process.

The particle size distribution is one of the most important characteristics of a latex, determining its rheological properties, maximum solid content, adhesion, drying time, etc. High solid content (HSC) latexes [1] are an excellent example of a product requiring an accurate control of the PSD. Their formulation usually requires a very welldefined PSD in order to maintain acceptable levels of viscosity. Some processes call for a bimodal PSD, where the distribution must contain a certain volume fraction of large and small particles, and where the ratio of the diameters of each population must be within set limits. The development of tools for PSD prediction is thus well motivated.

Emulsion polymerization is a very complex heterogeneous process involving a multitude of chemical and physical phenomena, many of which have not yet been completely elucidated (namely nucleation and coagulation). Moreover, first-principles mathematical models tend to be numerically intensive, especially those accounting for PSD. Regardless of these difficulties, significant progress has been made during the past decade in EP modeling, and particularly in modeling the PSD.

Models for EP can be classified in two levels according to the way they account for particle size [2]. Level-one models are based on the monodispersed approximation, i.e., they assume that all particles have the same average volume. Level-two models, on the other hand, account for the latex PSD by means of population balances. The type of model you choose depends on the system you have and the results you need. Models of the first level are still used at present, and will often provide useful results. However, in certain cases they are limited in nature because: i) the polymerization kinetics are only approximate, since the average number of radicals per particle is in general a non-linear function of the particle volume, and therefore a single average volume is not sufficient to describe the system; ii) systems with complex PSDs cannot be modeled. Level-two models suffer from none of these restrictions, being therefore the appropriate tool for modeling EP reactors when it is important to understand the dynamics of nucleation and 
growth, when simplifying assumptions are not acceptable, or in cases where one needs to account for differences in kinetics, composition, etc. as a function of particle size.

Some interesting reviews on the modeling of EP reactors have already been published. The first comprehensive discussion on the subject was given by Min and Ray [3], who presented a very general model framework including population balance equations to describe PSD and molecular weight distribution (MWD) in emulsion homopolymerization reactors. Saldívar et al. [4] did an excellent review on the modeling of emulsion copolymerization reactors, accounting for PSD but neglecting particle coagulation. Dubé et al. [5] also reviewed the modeling of copolymerization reactors, but on the basis of the monodispersed approximation. More recently, Gao and Penlidis [6] described a database/model for emulsion homo- and copolymerization, also based on the monodispersed approximation.

Since the subject of the present review is specifically the modeling of the PSD, the discussion will center on what distinguishes level-two from level-one models, for which updated reviews are available [5,6]. The fundamental difference between these two approaches is that level-two models include an additional transport equation: the population balance equation. Thus, we will look in detail at the formulation of full population balances for emulsion polymerization processes. Also, we will pay particular attention to the modeling of particle coagulation, given the importance of this phenomenon for the evolution of PSD. Finally, numerical methods for the solution of the governing equations will be discussed, since the accuracy and speed of the solution greatly determines the model applicability. 


\section{Formulation of the population balance}

The exact form of the population balance depends both on the type of process under study and on the modeling assumptions one necessarily has to make. Table 1 lists the population balance models (PBMs) developed during the last decade and specifies their major features: the type of reactor, the type of polymerization (homo/copolymerization), and the kinetic model assumed (e.g., the zero-one model where the particles are assumed to contain only either zero or one radical; pseudo-bulk where particles can have more than one radical, but the number of radicals is averaged over the total number of particles, etc.). In this section, we will review and discuss the formulation of population balances in a systematic manner, so as to cover the most frequent situations one can encounter when modeling EP processes.

\subsection{Principles of PBEs}

Some principles of the theory of population balance equations relevant for the subject of this review will be presented here. In particular, we will discuss the choice of internal coordinates, the formulation of the boundary condition (often incorrectly written in the literature), and some aspects of particle coagulation. For an interesting and detailed discussion on theory and application of population balances, the reader is referred to Ramkrishna [7].

Consider an open system where the particles are distributed according to their size, $x$, and position, $\mathbf{r}$, and let the domains of $x$ and $\mathbf{r}$ be represented by $\Omega_{x}$ and $\Omega_{\mathbf{r}}$. These two variables are also designated by internal and external coordinates, respectively. In addition, postulate that there exists an average number density function, $F(x, \mathbf{r}, t)$, such that $F(x, \mathbf{r}, t) d x d V_{\mathbf{r}}$ is the number of particles with size between $x$ and $x+d x$ in the infinitesimal volume $d V_{\mathbf{r}}$. Although this is not the most general case, it suffices for most applications in EP. The population balance for this density function is obtained through a number balance on the particles of state $(x, \mathbf{r})$, and can be shown to give [7]: 
$\frac{\partial F(x, \mathbf{r}, t)}{\partial t}=-\frac{\partial}{\partial x}(\& F(x, \mathbf{r}, t))-\nabla_{\mathbf{r}} \cdot(\mathbf{R} F(x, \mathbf{r}, t))+\mathfrak{R}(x, \mathbf{r}, t)$

Here, $\{(x, \mathbf{r}, t)$ is the rate of particle growth, $\boldsymbol{k}(x, \mathbf{r}, t)$ is the rate of change of the external coordinates, and $\mathfrak{R}(x, \mathbf{r}, t) d x d V_{\mathbf{r}}$ is the net rate of generation of particles with size between $x$ and $x+d x$ in the infinitesimal volume $d V_{\mathrm{r}}$. The first term on the RHS of Eq. (1) accounts for particle growth (i.e. motion through the internal property space), and the second term for particle transport (i.e. motion through physical space). The last term may include a variety of phenomena, namely particle formation and depletion due to coagulation. It may also account for nucleation, but as shown subsequently nucleation is usually treated through the boundary condition. If particle coagulation is included, $\Re(x, \mathbf{r}, t)$ will be a nonlinear functional of $F(x, \mathbf{r}, t)$, giving rise to an integrohyperbolic partial differential equation.

The PBE needs to be complemented with initial and boundary conditions. The initial condition (IC) must define the particle distribution, both in size and position. A typical example is,

$$
F(x, \mathbf{r}, 0)=N_{0} u(\mathbf{r}) w(x)
$$

where $N_{0}$ is the initial number of particles, and $u(\mathbf{r})$ and $w(x)$ are integral-normalized functions accounting respectively for their spatial and size distribution. The boundary condition (BC) will in turn account for the rate of particle nucleation. For example, if the new particles are formed with size $x_{\mathrm{nuc}}$ and the nucleation rate is $R_{\mathrm{nuc}}(\mathbf{r}, t)$, the $\mathrm{BC}$ is [7],

$$
F\left(x_{\mathrm{nuc}}, \mathbf{r}, t\right)=\frac{R_{\mathrm{nuc}}(\mathbf{r}, t)}{\mathfrak{x}\left(x_{\mathrm{nuc}}, \mathbf{r}, t\right)}
$$

since the particle flux at $x_{\text {nuc }}$ - given by $F\left(x_{\text {nuc }}, \mathbf{r}, t\right) \hat{x}\left(x_{\text {nuc }}, \mathbf{r}, t\right)$ - must equal the rate of nucleation. This is equivalent to the existence of a source term of the form $\delta\left(x-x_{\text {nuc }}\right) R_{\text {nuc }}(\mathbf{r}, t)$ on the RHS of Eq. (1). From the definition of $x_{\text {nuc }}$, it is clear that the domain of the internal coordinate must be $\Omega_{x} \equiv\left[x_{\text {nuc }}, \infty[\right.$. Though simple, this BC is 
well suited for EP. In fact, sensitivity analysis shows that, within reasonable limits, the value of $x_{\text {nuc }}$ has a negligible influence over the results [8]. Consequently, there is no need to define two distinct sizes and to account separately for particles formed by homogeneous and micellar nucleation.

The choice of the particle size for internal coordinate deserves a remark. Particle size is clearly the most intuitive choice when the objective is to compute the PSD, but there are alternatives, in particular, the birth time. Formulating the problem in terms of the birth time has the advantage of simplifying the PBE, because the divergence with respect to the internal coordinate becomes zero, but also the inconvenience of making it difficult to describe particle coagulation [9]. Therefore, in general, the particle size is the appropriate internal coordinate.

The equations presented above hold true irrespectively of the variable chosen as a measure of the particle size (radius, volume, mass, etc.). However, the derivation of the PBE is somewhat simpler when done in terms of the unswollen volume of the particles or the mass of polymer in the particles. This is because both the expression for the rate of particle growth and the coagulation kernels are easier to write in terms of these variables. Thus, in what follows, we will write the population balances in terms of the unswollen volume $(v)$. Nevertheless, equivalent relations can be written in terms other variables, namely the unswollen radius $(r)$, which is more convenient for the numerical solution of the equations. The relationship between the two density functions is given by:

$F_{r}(r, \mathbf{r}, t)=F_{v}(v, \mathbf{r}, t) \frac{d v}{d r}=4 \pi r^{2} F_{v}(v, \mathbf{r}, t)$

The different variables, swollen and unswollen, are trivially related by,

$v=\frac{4 \pi r^{3}}{3}=v_{\mathrm{s}} \phi_{\mathrm{pp}}=\frac{4 \pi r_{\mathrm{s}}^{3}}{3} \phi_{\mathrm{pp}}$

where $\phi_{\mathrm{pp}}$ is the volume fraction of polymer in the particles, and the index $s$ denotes the swollen property. 
When coagulation is the only phenomenon, apart from nucleation, contributing to the net particle generation rate, its value is just given by $\mathfrak{R}=\mathfrak{R}_{\text {coag }}^{+}-\mathfrak{R}_{\text {coag }}^{-}, v>v_{\text {nuc }}$. Here, the terms $\mathfrak{R}_{\text {coag }}^{+}$and $\mathfrak{R}_{\text {coag }}^{-}$account for particle formation and depletion due to coagulation, respectively. If we assume binary aggregation to be the dominant aggregation mechanism (i.e. no more than two particles aggregate simultaneously), it is possible to develop fairly simple expressions for the two coagulation terms [7],

$$
\mathfrak{R}_{\text {coag }}^{+}(v, \mathbf{r}, t)=\left\{\begin{array}{l}
0, \quad v \leq 2 v_{\text {nuc }} \\
\left\{\begin{array}{l}
\frac{1}{2} \int_{v_{\text {nuc }}}^{v-v_{\text {nuc }}} \beta\left(v^{\prime}, v-v^{\prime} ; \mathbf{r}, t\right) F\left(v^{\prime}, \mathbf{r}, t\right) F\left(v-v^{\prime}, \mathbf{r}, t\right) d v^{\prime} \text { or } \\
\int_{v_{\text {nuc }}}^{v / 2} \beta\left(v^{\prime}, v-v^{\prime} ; \mathbf{r}, t\right) F\left(v^{\prime}, \mathbf{r}, t\right) F\left(v-v^{\prime}, \mathbf{r}, t\right) d v^{\prime}, \quad v>2 v_{\text {nuc }}
\end{array}\right.
\end{array}\right.
$$

$$
\mathfrak{R}_{\text {coag }}^{-}(v, \mathbf{r}, t)=F(v, \mathbf{r}, t) \int_{v_{\text {nuc }}}^{\infty} \beta\left(v, v^{\prime} ; \mathbf{r}, t\right) F\left(v^{\prime}, \mathbf{r}, t\right) d v^{\prime}
$$

where $v_{\text {nuc }}$ is the unswollen volume of the nucleated particles (and also the lower bound of the internal coordinate domain), and $\beta\left(v, v^{\prime} ; \mathbf{r}, t\right)$ is the coagulation rate coefficient between particles of unswollen volume $v$ and $v^{\prime}$. The computation of $\beta$ is the object of Section 3. Concerning the details of Eq. (6), note that it is impossible to form particles with $v<2 v_{\text {nuc }}$ by coagulation. In addition, note that both expressions are equivalent; they are just different ways to avoid double counting the coagulation events. Finally, we underline that the boundary condition, Eq. (3), is not affected by the inclusion of coagulation [7, 10]. Unfortunately, there is a fair amount of confusion in the literature regarding this issue.

The assumption of binary aggregation implies dilute systems, where the probability of more than two particles aggregating at the same time is reduced $[7,11]$. Nevertheless, Eqs. (6) and (7) have been used irrespectively of the solid content of the latex. For example, this formulation was used at solid content of $55 \mathrm{wt} \%$ [12] and 20 wt\% [13]. Researchers working in other areas where PBEs are used seem to be facing the same problem (e.g. [14]). The reasons for this are most likely to be the extreme complexity of formulating and solving the equations for multi-body collisions, and the associated difficulty of identifying the parameters and experimentally validating the 
results. As will be shown bellow, this is a challenging task to do exactly for 2-body aggregation. Simply put, we are constrained to use this approximation by the impracticality of considering higher order phenomena.

\subsection{Kinetics}

\subsubsection{Introduction}

In an emulsion homopolymerization system, the latex particles can differ in size as well as in the number of radicals per particle, and in the degree of polymerization of these radicals. The growth rate of a particle obviously depends on the number of radicals, but is also affected by their degree of polymerization since the termination rate coefficient depends on the length of the terminating chains [15]. This is the so-called chain-length-dependent (CLD) termination. Because, in general, both the number and the degree of polymerization of the radicals affect the particle growth rate, such parameters should be included as additional internal coordinates in the PBE. Unfortunately, this leads to an intractable multidimensional PBE. The only way to overcome this difficulty is to reduce the dimensionality of the problem by making some approximations. The most common alternatives are:

- To neglect CLD termination. In this way we can remove the degree of polymerization of the radicals from the PBE.

- To neglect particles with two or more radicals: zero-one model. This automatically eliminates the problems related with termination and reduces the dimensionality of the equations since the number of radicals is limited to zero or one.

- To neglect compartmentalization [15] effects (i.e. the isolation of radicals in separate particles): pseudo-bulk model. The number of radicals is no longer an internal coordinate, since particles of the same size are assumed to have the same (average) number of radicals. One may or may not account for CLD termination, but in either case the chain length distribution of the growing chains does not appear as an internal coordinate in the PBE. 
Using the first hypothesis, we are still left with two internal coordinates: particle size and number of radicals per particle. This remains a complex problem, but Min and Ray [3] showed how to solve it by defining an infinite set of particle density functions, each accounting for the PSD of the particles having a given number of radicals. For a general case involving nucleation, coagulation, variable coefficients, etc., the infinite set of integro-hyperbolic partial differential equations given by Min and Ray can only be solved by numerical methods, at the expense of large computation times. Consequently, this approach (even if approximate) is rarely used to compute the PSD. In fact, to the best of our knowledge, only Min and Ray [16] made use of it. For special cases, analytic solutions are available. In particular, Giannetti derived a solution applicable to the Interval II of emulsion polymerization (no nucleation, no coagulation, constant coefficients, etc.) and later extended it to the Interval I [17, 18]. This kind of solutions may be used to gain insight into system behavior and to check numerical results, but they are of limited interest for reactor simulation.

The other two alternatives to handle the kinetics, i.e. the zero-one and the pseudo-bulk model, are substantially simpler and thus widely used in EP models (see Table 1). These will be reviewed in the following. For simplicity, the PBEs are presented for ideal stirred tank reactors (STRs) (batch, semi-batch and continuous). Generalizations to multicomponent systems and to other types of reactors are the subject of Sections 2.3 and 2.4, respectively.

\subsubsection{Zero-one model}

In a zero-one system, the entry of a radical into a particle already containing a radical causes termination at a rate much higher than that of the overall polymerization [19]. Therefore, a particle can have either zero or one radicals. For zero-one systems, it becomes feasible to use an exact mathematical formulation because: i) the number of PBEs is reduced given that $n=\{0,1\}$; ii) termination does not have to be taken into consideration.

There are two alternative treatments for zero-one systems. One possibility is to distinguish only between particles having zero or one radicals [15, 20]. Another possibility, more frequently used [8, 19, 21, 22], consists in further dividing particles having one radical in two populations, according to whether the radical is monomeric or 
polymeric. Since only monomeric radicals are assumed to desorb, this facilitates the description of radical desorption [23-25]. For an ideal STR, the corresponding PBEs are,

$$
\begin{aligned}
& \frac{1}{V_{\mathrm{w}}} \frac{\partial}{\partial t}\left(V_{\text {w }} f_{0}(v, t)\right)=\rho\left(f_{1}(v, t)-f_{0}(v, t)\right)+k_{\mathrm{dM}} f_{1 \mathrm{M}}(v, t) \\
& \quad+\frac{1}{2} \int_{v_{\text {nuс }}}^{v-v_{\text {nuc }}} \beta\left(v-v^{\prime}, v^{\prime} ; t\right)\left[f_{0}\left(v-v^{\prime}, t\right) f_{0}\left(v^{\prime}, t\right)+f_{1}\left(v-v^{\prime}, t\right) f_{1}\left(v^{\prime}, t\right)\right] d v^{\prime} \\
& \quad-f_{0}(v, t) \int_{v_{\text {muc }}}^{\infty} \beta\left(v, v^{\prime} ; t\right) F\left(v^{\prime}, t\right) d v^{\prime}+\frac{Q^{\text {in }} \phi_{\mathrm{w}}^{\text {in }}}{V_{\text {w }}} f_{0}^{\text {in }}(v, t)-\frac{Q^{\text {out }} \phi_{\mathrm{w}}^{\text {out }}}{V_{\mathrm{w}}} f_{0}(v, t)
\end{aligned}
$$

$$
\begin{gathered}
\frac{1}{V_{\mathrm{w}}} \frac{\partial}{\partial t}\left(V_{\mathrm{w}} f_{1 \mathrm{P}}(v, t)\right)=-\frac{\partial}{\partial v}\left(K(v, t) f_{1 \mathrm{P}}(v, t)\right)+\rho_{\mathrm{I}} f_{0}(v, t)-\rho f_{1 \mathrm{P}}(v, t)-k_{\mathrm{fM}}[\mathrm{M}]_{\mathrm{p}} f_{1 \mathrm{P}}(v, t) \\
+k_{\mathrm{p}}[\mathrm{M}]_{\mathrm{p}} f_{1 \mathrm{M}}(v, t)+\int_{v_{\text {nuc }}}^{v-v_{\text {nuc }}} \beta\left(v-v^{\prime}, v^{\prime} ; t\right) f_{0}\left(v-v^{\prime}, t\right) f_{1 \mathrm{P}}\left(v^{\prime}, t\right) d v^{\prime} \\
-f_{1 \mathrm{P}}(v, t) \int_{v_{\text {nuc }}}^{\infty} \beta\left(v, v^{\prime} ; t\right) F\left(v^{\prime}, t\right) d v^{\prime}+\frac{Q^{\text {in }} \phi_{\mathrm{w}}^{\text {in }}}{V_{\mathrm{w}}} f_{1 \mathrm{P}}^{\text {in }}(v, t)-\frac{Q^{\text {out }} \phi_{\mathrm{w}}^{\text {out }}}{V_{\mathrm{w}}} f_{1 \mathrm{P}}(v, t)
\end{gathered}
$$

$$
\begin{gathered}
\frac{1}{V_{\mathrm{w}}} \frac{\partial}{\partial t}\left(V_{\mathrm{w}} f_{1 \mathrm{M}}(v, t)\right)=\rho_{\mathrm{E}} f_{0}(v, t)-\left(\rho+k_{\mathrm{dM}}\right) f_{1 \mathrm{M}}(v, t)+k_{\mathrm{fM}}[\mathrm{M}]_{\mathrm{p}} f_{1 \mathrm{P}}(v, t) \\
-k_{\mathrm{p}}[\mathrm{M}]_{\mathrm{p}} f_{1 \mathrm{M}}(v, t)+\int_{v_{\text {nuc }}}^{v-v_{\text {muc }}} \beta\left(v-v^{\prime}, v^{\prime} ; t\right) f_{0}\left(v-v^{\prime}, t\right) f_{1 \mathrm{M}}\left(v^{\prime}, t\right) d v^{\prime} \\
-f_{1 \mathrm{M}}(v, t) \int_{v_{\text {nuc }}}^{\infty} \beta\left(v, v^{\prime} ; t\right) F\left(v^{\prime}, t\right) d v^{\prime}+\frac{Q^{\text {in }} \phi_{\mathrm{w}}^{\text {in }}}{V_{\mathrm{w}}} f_{1 \mathrm{M}}^{\text {in }}(v, t)-\frac{Q^{\text {out }} \phi_{\mathrm{w}}^{\text {out }}}{V_{\mathrm{w}}} f_{1 \mathrm{M}}(v, t)
\end{gathered}
$$

where $\rho$ is the total entry frequency, $\rho_{I}$ is the entry frequency of initiator-derived (polymeric) radicals, $\rho_{\mathrm{E}}$ is the entry frequency of monomeric radicals, $k_{\mathrm{dM}}$ is the rate coefficient for desorption of monomeric radicals from particles, and $k_{\mathrm{fM}}$ is the rate coefficient for transfer to monomer. The density functions of the particles containing zero radicals, one monomeric radical and one polymeric radical are, respectively, $f_{0}(v, t), f_{1 \mathrm{M}}(v, t)$ and $f_{1 \mathrm{P}}(v, t)$. By definition, $f_{1}=f_{1 \mathrm{M}}+f_{1 \mathrm{P}}$ and $F=f_{0}+f_{1}$. The reader is referred to Nomenclature for the meaning of the remaining variables. For a homopolymerization system, the volume growth rate of a particle containing one radical is given by the well-known expression: 
$\varepsilon(v, t)=K(v, t)=\frac{k_{\mathrm{p}} M W_{\mathrm{m}}[\mathrm{M}]_{\mathrm{p}}}{N_{\mathrm{A}} \rho_{\mathrm{p}}}$

Notice that, in general, $K$ will be a function of particle size and time. As a simplification, the quasi-steady state assumption can be applied to Eq. (10), and the coagulation and flow terms neglected in order to obtain an algebraic expression for $f_{1 \mathrm{M}}(v, t)$,

$f_{1 \mathrm{M}}(v, t)=\frac{k_{\mathrm{fM}}[\mathrm{M}]_{\mathrm{p}} f_{1 \mathrm{P}}(v, t)+\rho_{\mathrm{E}} f_{0}(v, t)}{k_{\mathrm{p}}[\mathrm{M}]_{\mathrm{p}}+k_{\mathrm{dM}}+\rho}$

Gilbert [19] and Coen et al. [8, 21] were the first to present the PBEs (including coagulation) for this version of the zero-one model. However, note that in these works the terms accounting for particle formation due to coagulation were incorrectly transcribed in press (although the authors have affirmed that the correct formulation was used in the simulations [26]). These PBEs can easily be derived if we recognize that, termination being instantaneous, both the entry of a radical into a particle already containing a radical and the coagulation between two active particles lead to a dead particle. Concerning the coagulation terms, note that the factor $1 / 2$ must only be included in the rate of particle formation due to coagulation when the same particle density function appears twice in the kernel (to avoid double counting). A good way to check these PBEs for consistency is to add them so as to derive a population balance for $F(v, t)$. We must obtain the global population balance expressed in the next section by Eq. (15), with $\bar{n}(v, t)=f_{1}(v, t) / F(v, t)$.

The initial and boundary conditions can be easily deduced from Eqs. (2) and (3). The ICs are,

$\left\{\begin{array}{l}f_{0}(v, 0)=\left(N_{0} / V_{\mathrm{w}}\right) w(v) \\ f_{1 \mathrm{M}}(v, 0)=0 \\ f_{1 \mathrm{P}}(v, 0)=0\end{array}\right.$ 
since seed particles, if present, do not contain radicals. If we suppose that new particles always contain one polymeric radical (entry of $i$-mers into micelles, with $z \leq i<j_{\text {crit }}$, or

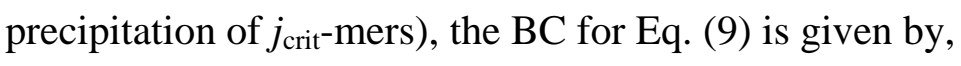

$$
f_{1 \mathrm{P}}\left(v_{\mathrm{nuc}}, t\right)=\frac{R_{\mathrm{nuc}}(t)}{K\left(v_{\mathrm{nuc}}, t\right)}
$$

where $R_{\text {nuc }}(t)$ is the total (micellar and homogenous) nucleation rate.

In order to use these equations it is essential to determine under which conditions the zero-one model can be used to describe the PSD. Theoretical and experimental tests to check if a given system obeys zero-one kinetics have been developed by Gilbert and co-workers [15, 24, 27, 28]. For example, a necessary but not sufficient condition is that $\bar{n} \leq 0.5$. Nevertheless, it is necessary to make a clear distinction between the applicability of the zero-one model for the prediction of the kinetic behavior of the system (i.e. $\bar{n}$ ) and the prediction of PSD. This problem was discussed in detail by Giannetti [17] and is of primary importance for the modeling of PSD. According to this author, even if the contribution of particles with more than one radical is negligible for the kinetic behavior of the system when $\bar{n}<<0.5$, the same is not true for the PSD. Actually, the fact that particles with more than one radical do exist, increases the broadness of the PSD with respect to that predicted by the zero-one model. This is a shortcoming to bear in mind when using this approach to compute the PSD.

Since this approach is only suitable for systems following zero-one kinetics, its applicability is somewhat limited. In fact, zero-one systems generally concern small particles (for which the rate of radical entry is low and the rates of radical desorption and termination are high) and low conversions (as to avoid a significant Trommsdorff effect). Despite its inherent limitations, the zero-one model can be of great value in interpreting mechanistic studies. In particular, it has been used by Gilbert's workgroup to investigate the mechanisms of particle formation in the emulsion polymerization of styrene $[8,15,19]$. This approach has also been used in a few reaction engineering studies. Zeaiter et al. [29] extended Coen et al.'s model [8] to predict the evolution of PSD and MWD during the semi-batch emulsion polymerization of styrene. The model was used to study the possibility of controlling PSD and MWD by manipulation of the 
reactor temperature and monomer flow rate. Crowley et al. [30] simplified the model proposed by Coen et al., by neglecting particle coagulation, and used it to investigate the optimization of the surfactant feed profile given a target bimodal PSD. Meadows et al. [31] extended the model by Coen et al. to non-isothermal conditions, neglecting particle coagulation, and used it to explore methods for controlling the PSD.

\subsubsection{Pseudo-bulk model}

As mentioned in Section 2.2.1, an alternative approximation is to average the number of radicals over all particles of size $v$, and define an average growth rate $\mathfrak{k}(v, t)=K(v, t) \bar{n}(v, t)$. This leads to the pseudo-bulk (PB) model. On the basis of this approximation, the PBE for an ideal STR can be derived without difficulty from the principles exposed in Section 2.1,

$$
\begin{aligned}
\frac{1}{V_{\mathrm{w}}} \frac{\partial}{\partial t}\left(V_{\mathrm{w}}\right. & F(v, t))=-\frac{\partial}{\partial v}(\mathcal{v}(v, t) F(v, t)) \\
& +\frac{1}{2} \int_{v_{\text {nuc }}}^{v-v_{\text {nuc }}} \beta\left(v^{\prime}, v-v^{\prime} ; t\right) F\left(v^{\prime}, t\right) F\left(v-v^{\prime}, t\right) d v^{\prime}-F(v, t) \int_{v_{\text {nuc }}}^{\infty} \beta\left(v, v^{\prime} ; t\right) F\left(v^{\prime}, t\right) d v^{\prime} \\
& +\frac{Q^{\text {in }} \phi_{\mathrm{w}}^{\text {in }}}{V_{\mathrm{w}}} F^{\text {in }}(v, t)-\frac{Q^{\text {out }} \phi_{\mathrm{w}}^{\text {out }}}{V_{\mathrm{w}}} F(v, t)+\delta\left(v-v_{\text {nuc }}\right) R_{\text {nuc }}(t)
\end{aligned}
$$

Since this approximation decouples $F(v, t)$ from the distribution of the number of radicals, an expression for $\bar{n}(v, t)$ must also be supplied (see Section 2.2.4). The initial and boundary conditions can be easily deduced from Eqs. (2) and (3),

$$
\begin{aligned}
& F(v, 0)=\left(N_{0} / V_{\mathrm{w}}\right) w(v) \\
& F\left(v_{\mathrm{nuc}}, t\right)=\frac{R_{\mathrm{nuc}}(t)}{\mathfrak{k}\left(v_{\mathrm{nuc}}, t\right)}
\end{aligned}
$$

The validity of the PB model is conditioned by the assumption of uniform growth. In general, this hypothesis only holds true for high values of $\bar{n}(v, t)$ (the radical 
distribution among particles is then sufficiently uniform to validate the averaging procedure) or for high radical entry rates. Note that the limits of applicability of Eq. (15) are not to be confused with those of the pseudo-bulk equation for $\bar{n}$ (see Section 2.2.4). If the condition of uniform growth is not satisfied, then particles with $n<\bar{n}$ will lag behind and particles with $n>\bar{n}$ will grow more quickly. This will give rise to what is called stochastic broadening [15], a phenomena not taken into account by the PB approach. Accordingly, the accuracy of the results obtained with the PB model will depend on the relative importance of stochastic broadening. For example, stochastic broadening will not be of importance for CSTRs since the PSD is inherently broad [9, 32].

Despite this shortcoming, the compromise between simplicity and predictability appears to have favored the generalized use of the PB approach for modeling PSD in EP reactors, as seen in Table 1. This is probably because: i) the PB model has the merit of reducing the computation of $F(v, t)$ to the solution of a single PBE; ii) unlike the zeroone model, the PB model presents no restrictions with regards to the maximum number of radicals per particle, thus allowing one to simulate the entire conversion range; iii) stochastic broadening is perhaps comparable in significance to other phenomena not accounted for, or accounted for in approximate ways (e.g. coagulation).

A somewhat different method has recently been proposed by Coen et al. [22]. The purpose was to extend the previous model by Coen et al. [8] to systems where pseudo-bulk kinetics are important during the nucleation period. The authors defined a cross-over radius $r_{\mathrm{co}}$ and used it to divide the particle size domain in two regions: zeroone kinetics for $r<r_{\text {co }}$, and pseudo-bulk kinetics for $r>r_{\text {co }}$. To simplify the mathematics, the authors did not include coagulation above $r_{\mathrm{co}}$. However, this assumption seems questionable, as there is no reason to believe that the coagulation rate of small-big particles is insignificant with respect to that of small-small particles. The equations presented in Section 2.2.2 were employed to describe the zero-one region, while the PB domain was described by Eq. (15) without the coagulation terms. The effect of CLD termination was included in the computation of $\bar{n}(v, t)$, as described in Section 2.2.4. The authors do not mention how they linked the two PBEs; in particular, it is not clear if the formation of particles with $r>r_{\text {co }}$ by coagulation of two particles with $r<r_{\text {co }}$ was taken into account. This approach was applied to the ab initio polymerization of butyl acrylate. The evolution of conversion and the effects of initiator 
and surfactant concentration on the final particle number were correctly predicted, but no comparison was made with experimental measures of the PSD.

Combining the zero-one and PB models (hybrid model) seems an interesting way to overcome some limitations of the individual methods. Small particles in the size range where termination is not rate-determining are best described by the zero-one model. The remaining particles are described by the PB model.

\subsubsection{Average number of radicals per particle}

The PB model requires an expression for the average number of radicals in particles of size $v, \bar{n}(v, t)$. There are basically two ways of deriving approximate equations for this quantity: i) to account for compartmentalization, while neglecting CLD termination; ii) to account for CLD termination, while ignoring compartmentalization.

The first method is used almost exclusively in the field of polymer reaction engineering, but is prone to contestation. First, because there is strong evidence that CLD termination cannot be neglected [33, 34]. Second, because Eq. (15) is only valid for conditions where compartmentalization is not significant. Hence, one could question the usefulness of an equation for $\bar{n}(v, t)$ that takes compartmentalization into account. The justification for this choice is that the PB model is frequently used to describe the evolution of PSD outside its limits of validity, where compartmentalization effects may indeed be relevant. In addition, for reactor modeling it is usually found that an "average" value of the termination rate coefficient is sufficient to reproduce the experimental results.

If CLD termination is neglected, an approximate solution can be found by solving a simplified form of the infinite set of density functions mentioned in Section 2.2.1 [4, 9]. Assuming that radical entry, desorption and termination are much faster than coagulation, growth and inflow/outflow, we obtain a set of PBEs which is simply a slightly modified version of the Smith-Ewart differential equations [35]. If we further apply the quasi-steady-state assumption, the steady-state value of $\bar{n}(v, t)$ can be determined analytically from the Stockmayer-O'Toole solution [36], 


$$
\bar{n}(v, t)=\frac{a}{4} \frac{I_{m}(a)}{I_{m-1}(a)}
$$

where $a(v, t)$ and $m(v, t)$ are, respectively, the argument and order of the modified Bessel function of the first kind $I_{m}(a)$. These two parameters are given by,

$$
a=(8 \alpha)^{1 / 2}=\left(\frac{8 \rho}{c}\right)^{1 / 2}
$$

$$
m=\frac{k_{\mathrm{des}}}{c}
$$

where $k_{\mathrm{des}}$ is the desorption frequency [25], and $c\left(k_{\mathrm{t}} / N_{\mathrm{A}} v_{\mathrm{s}}\right)$ is the pseudo-first-order rate coefficient for termination. We stress that $\rho$ in Eq. (19) is the total entry frequency of radicals into particles, given by $\rho=\rho_{I}+\rho_{E}$.

Usually, to avoid the computation of the modified Bessel functions in Eq. (18), the partial fraction expansion proposed by Ugelstad et al. [37] is employed,

$$
\bar{n}(v, t)=\frac{\alpha}{m+\frac{2 \alpha}{m+1+\frac{2 \alpha}{m+2+\frac{2 \alpha}{m+3+\ldots}}}}
$$

According to Dubé et al. [5], approximately ten levels of fractions are necessary to obtain convergence. Notice, however, that the number of levels required increases with the value of $\bar{n}(v, t)$, and should preferably be determined by trial and error for the particular application.

More recently, Li and Brooks [38] proposed a semi-theoretical expression for determining both the time-dependent and steady state values of the average number of radicals. The steady-state solution is particularly interesting, as it compares very well with Eq. (18) and is explicit: 


$$
\bar{n}(v, t)=\frac{2 \alpha}{m+\sqrt{m^{2}+8 \alpha \frac{2 \alpha+m}{2 \alpha+m+1}}}
$$

The relative error of the $\bar{n}(v, t)$ values obtained by this expression with respect to Eq. (18) is shown in Fig. 1. The difference is seen to be at most 4\%, demonstrating that Eq. (22) might be a good alternative to the more time-consuming Eqs. (18) and (21).

Another option to compute the average number of radicals per particle is the pseudo-bulk equation [15]:

$$
\frac{\partial \bar{n}(v, t)}{\partial t}=\rho-k_{\mathrm{des}} \bar{n}(v, t)-2 c \bar{n}(v, t)^{2}
$$

This expression results from a further simplification of the modified Smith-Ewart equations mentioned above. As a result, this equation is only valid for $\bar{n}>0.7$ or for $c<<, k_{\text {des }}[15,39]$.

The inclusion of chain-length dependency in the Smith-Ewart equations is rather difficult, although not impossible [39]. Nevertheless, for pseudo-bulk systems, where compartmentalization effects are not important, a mechanistic approach is feasible. Russell et al. [40] addressed this issue in detail; thus only the main features of the treatment will be outlined here. The first step is to establish the balances for the chain distribution $\left\{R_{i}\right\}$, where $R_{i}$ denotes the average number of growing chains of length $i$ per particle. This requires a physical model for the termination rate coefficient between chains of length $i$ and $j, k_{\mathrm{t}}^{i j}$. Then, one of several approximations available may be used to compute $\left\{R_{i}\right\}$, from which $\bar{n}(v, t)$ can be determined. A formal average termination rate coefficient can also be defined:

$$
\left\langle k_{\mathrm{t}}\right\rangle=\frac{\sum_{i, j} k_{\mathrm{t}}^{i j} R_{i} R_{j}}{\left(\sum_{i} R_{i}\right)^{2}}
$$

In particular, this average value may be used together with Eq. (23) to compute $\bar{n}(v, t)$. 
The choice between these two approaches is dictated by the kinetics of the system, the modeling objectives, the level of complexity desired and the available data (diffusion coefficients, etc.).

\subsection{Multicomponent systems}

\subsubsection{Pseudo-homopolymerization approach}

Given the practical importance of emulsion copolymerization, it is natural that one wishes to extend the methods developed for the modeling of PSD in homopolymerization systems to copolymerization reactors. The PBEs governing multicomponent systems are far more complex than those of homopolymer systems (see Section 2.2.1), as particles can have radicals with different end groups (and composition). The dimensionality of the problem is thus superior. Fortunately, under certain conditions, such multivariate PBEs can be reduced to the homopolymer equations described in the foregoing sections by use of the so-called pseudohomopolymerization approach [4, 41-43]. Thanks to this treatment, the only difference is that some parameters are replaced by proper averages.

In particular, the PB model described in Section 2.2.3 is extensively employed in the modeling of multicomponent systems (see Table 1). Saldívar et al. [4] reviewed in detail the modeling of copolymerization reactors, and especially the use of this approach for computing the evolution of PSD in the absence of coagulation. The simulation of a multicomponent system requires a number of additional relations in order to account for comonomer partition, comonomer conservation, etc. The PBE, however, is the same as for a homopolymer system: Eq. (15). The only difference concerns the growth rate, which is given by the following average [4],

$$
\kappa(v, t)=\frac{\bar{n}(v, t)}{N_{\mathrm{A}}} \sum_{i, j=1}^{c} \frac{k_{\mathrm{p} i j} p_{i}\left[\mathrm{M}_{j}\right]_{\mathrm{p}} M W_{j}}{\rho_{\mathrm{p} j}}
$$


where $p_{i}$ is the probability of a particle having a radical ending in monomer $i$. The value of $\bar{n}(v, t)$ can be determined from Eqs. (18)-(23), with appropriate average coefficients [4].

Asúa's workgroup used this approach to model the evolution of PSD and conversion in the emulsion copolymerization of vinyl acetate and Veova 10 in a continuous loop reactor $[12,44,45]$. Good agreement was achieved between experimental and computed PSDs. As the reactivity ratios of these monomers are close to unity, copolymer composition was not an issue. It is worth pointing out that this type of process specifically calls for a population balance approach because of the broad PSDs obtained. Saldívar et al. [4, 46] used their comprehensive model to simulate various binary systems and polymerization strategies. In particular, they validated their model against experimental data on the copolymerization of styrene and methyl methacrylate in batch and continuous stirred tank reactors. The model was capable of predicting the effect of a number of variables on conversion, average particle diameter and copolymer composition. Unfortunately, no comparison with experimental PSDs was made. Immanuel et al. [13, 47] developed two models for the semi-batch emulsion copolymerization of vinyl acetate and butyl acrylate: a coagulation-free and a coagulation-inclusive model. The latter model was shown to provide better predictions of the PSD, but only at a qualitative level. Kammona et al. [48] modeled the emulsion copolymerization of styrene and 2-ethylhexyl acrylate. The simulations compared successfully with experimental data on overall conversion and average particle diameter at different monomer, initiator and emulsifier concentrations. An example of comparison between experimental and predicted PSDs was given, showing good agreement.

\subsubsection{Multicomponent approach}

An important limitation of Eq. (15) is that it contains no information on the composition of the particles (the only internal coordinate is the particle size). As a result, when such a PBE is employed for the modeling of multicomponent systems, it is implicitly assumed that every latex particle has the same polymer composition. Even if convenient, this simplifying hypothesis is only a plausible approximation for systems comprised of particles with similar histories. For instance, one may expect this approach 
to be valid for the modeling of the $a b$ initio synthesis of a monodispersed latex. Examples of systems for which the uniform composition hypothesis will not be applicable can also provided. Consider a semi-batch process involving two monomers with distinct reactivity ratios where a second population of particles is formed by secondary nucleation. It is clear that, unless some special feed policy is used, the new particles will be richer in the less reactive monomer. As a second example, suppose a train of two CSTRs operating in steady-state. If the comonomers have different reactivity ratios and no supplemental feed is added to the second reactor, the final latex will contain particles of the same size and different composition [32].

Particle composition can play a key role in the evolution of PSD. In fact, particle composition may affect the monomer concentrations in the particle, which in turn can have an effect upon the particle growth rate. In addition, the composition of the particles can also affect their colloidal stability (e.g. surface hydrophilicity, surfactant adsorption, Hamaker constant, etc.), and thus the coagulation rate. To take these effects into consideration, a PBE that explicitly includes particle composition in the particle state vector must be used. In what follows, we will illustrate this procedure by deriving an extended PBE that accounts for the cumulative composition of the polymer in the particles. Of course, more general treatments are possible.

The set of internal coordinates used to define the particle state in the extended PBE must be chosen in such way that one can determine univocally the amount of each type of polymer in the particles. For convenience, we will define and use two massbased particle state vectors (see Section 2.1). Let $m$ be the total mass of polymer in a given particle, $m_{i}$ the mass of polymer of type $i$ in the particle, and $w_{i}=m_{i} / m$ the mass fraction of polymer of type $i$ in the particle. Based on these quantities, we can define the vector of polymer masses, $\mathbf{m}=\left(m_{1}, m_{2}, \ldots, m_{c}\right)$, and the vector of mass fractions $\mathbf{w}=\left(w_{1}, w_{2}, \ldots, w_{c-1}\right)$, where $c$ is the number of comonomers. Clearly, the particle state can be determined from $\mathbf{m}$ or, alternatively, from $(m, \mathbf{w})$.

Let us now proceed to the definition of the density functions. We postulate that there exists a number density function $F_{\mathbf{m}}(\mathbf{m}, t)$, such that $F_{\mathbf{m}}(\mathbf{m}, t) d m_{1} d m_{2} \ldots d m_{c}$ is the number of particles per unit volume of aqueous phase with state $\mathbf{m}$ at time $t$. In the same way, we define $F_{\mathbf{w}}(m, \mathbf{w}, t)$, such that $F_{\mathbf{w}}(m, \mathbf{w}, t) d m d w_{1} d w_{2} \ldots d w_{c-1}$ is the number of particles per unit volume of aqueous phase with state $(m, \mathbf{w})$ at time $t$. The relation 
between these two density functions can be obtained from the Jacobian of the transformation $\mathbf{m}=\mathbf{m}(m, \mathbf{w})$ :

$$
F_{\mathbf{m}}(\mathbf{m}, t) m^{c-1}=F_{\mathbf{w}}(m, \mathbf{w}, t)
$$

The definition of two density functions is not of necessity, but it is rather useful. The $\mathrm{PBE}$ is easier to derive in terms of $F_{\mathbf{m}}(\mathbf{m}, t)$, since the expressions for the growth and coagulation terms are much simpler in terms of $\mathbf{m}$. On the other hand, $F_{\mathbf{w}}(m, \mathbf{w}, t)$ is best suited for computing the relevant properties of the dispersed phase. For example, the PSD is simply given by:

$$
F(m, t)=\int_{0<w_{i}<1} F_{\mathbf{w}}(m, \mathbf{w}, t) d w_{1} d w_{2} \ldots d w_{c-1}
$$

The PBE for the number density function $F_{\mathbf{m}}(\mathbf{m}, t)$ can be derived from the more general version of Eq. (1) (see, for instance, Ramkrishna [7]). For an ideal STR it reads:

$$
\begin{aligned}
\frac{1}{V_{\mathrm{w}}} \frac{\partial}{\partial t}\left(V_{\mathrm{w}}\right. & \left.F_{\mathbf{m}}(\mathbf{m}, t)\right)=-\sum_{i=1}^{c} \frac{\partial}{\partial m_{i}}\left(h \mathbf{\alpha}(\mathbf{m}, t) F_{\mathbf{m}}(\mathbf{m}, t)\right) \\
& +\frac{1}{2} \int_{\mathbf{\Omega}_{\mathbf{m}}} \beta\left(\mathbf{m}^{\prime}, \mathbf{m}-\mathbf{m}^{\prime} ; t\right) F_{\mathbf{m}}\left(\mathbf{m}^{\prime}, t\right) F_{\mathbf{m}}\left(\mathbf{m}-\mathbf{m}^{\prime}, t\right) d m_{1}{ }^{\prime} d m_{2}{ }^{\prime} \ldots d m_{c}{ }^{\prime} \\
& -F_{\mathbf{m}}(\mathbf{m}, t) \int_{\boldsymbol{\Omega}_{\mathrm{m}}} \beta\left(\mathbf{m}, \mathbf{m}^{\prime} ; t\right) F_{\mathbf{m}}\left(\mathbf{m}^{\prime}, t\right) d m_{1}{ }^{\prime} d m_{2}{ }^{\prime} \ldots d m_{c}{ }^{\prime} \\
& +\frac{Q^{\text {in }} \phi_{\mathrm{w}}^{\text {in }}}{V_{\mathrm{w}}} F_{\mathbf{m}}^{\text {in }}(\mathbf{m}, t)-\frac{Q^{\text {out }} \phi_{\mathrm{w}}^{\text {out }}}{V_{\mathrm{w}}} F_{\mathbf{m}}(\mathbf{m}, t)+\delta\left(\mathbf{m}-\mathbf{m}_{\mathrm{nuc}}\right) R_{\mathrm{nuc}}(t)
\end{aligned}
$$

Here, $\boldsymbol{\Omega}_{\mathbf{m}}$ is the domain of the particle state, and $\mathbf{m}_{\mathbf{n u c}}$ is a vector describing the mass of each type of polymer in the nucleated particles. This is the multicomponent equivalent of the one-dimensional Eq. (15). For the chosen particle state vector, the rate of change of the internal coordinates simply corresponds to the rate of production of polymer of type $i$ in the particle, which may be computed from the standard expression [4]: 
$n \underset{l}{\alpha}(\mathbf{m}, t)=\sum_{j=1}^{c} k_{\mathrm{p} j i} \frac{\bar{n} p_{j}}{N_{\mathrm{A}}}\left[\mathrm{M}_{i}\right]_{\mathrm{p}} M W_{i}$

The coagulation rate coefficient, $\beta\left(\mathbf{m}, \mathbf{m}^{\prime}\right)$, is computed by the usual procedure. However, it may now include explicit dependency on particle composition.

To the best of our knowledge, the application of this approach to modeling EP reactors has not yet been published.

\subsection{Other types of reactors}

Aside from the very common stirred tank reactors (batch, semi-batch and continuous), tubular reactors are also used for emulsion polymerization. In addition, non-ideal behavior (i.e. imperfect mixing) may in certain cases affect significantly the performance of stirred tank reactors $[49,50]$. Here, we will review the modeling of the PSD for these types of reactors. All equations presented in this section are based on the one-dimensional PB approach, given its widespread use. Extension to the zero-one model should pose no major problem.

\subsubsection{Tubular reactors}

Continuous tubular reactors have also been used for emulsion polymerization, though to a lesser extent than semi-batch reactors and CSTRs [32, 51]. A wide range of reactors fall into this category, namely: single-pass reactors, continuous loop reactors, packed beds, etc.

A key point in modeling a tubular reactor is the description of the flow pattern. Depending on the flow regime and reactor geometry, two distinct models have been used to describe the flow pattern in emulsion tubular reactors [52]: i) laminar flow with radial dispersion; ii) one-dimensional flow with axial dispersion. Usually, the residence time distribution (RTD) is determined experimentally and the model that best describes the results is applied $[45,51,52]$. Paquet and Ray [52] mention that the axial dispersion model is applicable to a wider range of reactors. 
It is worth noting that a continuous loop reactor [53] operating at high recycle ratios has a RTD similar to that of an ideal CSTR. If this is the case, the non-distributed equations presented in Section 2.2 may be used to compute the PSD, with much less computational effort. For example, Asúa and co-workers [12, 44] made use of this approximation in developing a PSD model for emulsion copolymerization in a loop reactor, after having measured its RTD.

Once the model for the flow pattern is chosen, it can be combined with Eq. (1) to obtain the required PBE. We will illustrate how this may be done for the axial dispersion model, which is the most frequently used. Using the PB approach, we can take Eq. (1) and write (with $\mathbf{r} \equiv z$ ),

$$
\begin{aligned}
\frac{\partial F(v, z, t)}{\partial t}= & -\frac{\partial}{\partial v}(\mathcal{L}(v, z, t) F(v, z, t))-\frac{\partial}{\partial z}(\delta F(v, z, t)) \\
& +\mathfrak{R}_{\text {coag }}^{+}-\mathfrak{R}_{\text {coag }}^{-}+\delta\left(v-v_{\text {nuc }}\right) R_{\text {nuc }}(z, t)
\end{aligned}
$$

where $z$ is the length coordinate of the reactor, and $\xi$ is the velocity of the particles in the axial direction. The terms $\mathfrak{R}_{\text {coag }}^{+}$and $\mathfrak{R}_{\text {coag }}^{-}$are given by Eqs. (6) and (7). The particle transport term can now be divided in two components, accounting independently for transport by convection and dispersion,

$$
\begin{aligned}
\frac{\partial F(v, z, t)}{\partial t}= & -\frac{\partial}{\partial v}(\delta(v, z, t) F(v, z, t))-\frac{\partial}{\partial z}\left(u_{z} F(v, z, t)\right)+D \frac{\partial^{2} F(v, z, t)}{\partial z^{2}} \\
& +\Re_{\text {coag }}^{+}-\Re_{\text {coag }}^{-}+\delta\left(v-v_{\text {nuc }}\right) R_{\text {nuc }}(z, t)
\end{aligned}
$$

where $u_{z}$ is the average axial velocity, and $D$ is the axial dispersion coefficient (assumed to be constant).

The IC is given by Eq. (2). As before, the BC with respect to the internal coordinate $(v)$ is given by,

$$
F\left(v_{\text {nuc }}, z, t\right)=\frac{R_{\text {nuc }}(z, t)}{\mathfrak{k}\left(v_{\text {nuc }}, z, t\right)}
$$


Because the differential equation above is also second order with respect to $z$, two additional BCs are required. These may be given by the Danckwerts conditions (the reactor is assumed to be closed),

$$
\begin{aligned}
& F\left(v, 0^{-}, t\right)=F\left(v, 0^{+}, t\right)-\left.\frac{D}{u_{z}} \frac{\partial F(v, z, t)}{\partial z}\right|_{z=0^{+}} \\
& \left.\frac{\partial F(v, z, t)}{\partial z}\right|_{z=L}=0
\end{aligned}
$$

where $L$ is the length of the reactor. Other BCs are possible for the axial dispersion model; see for example Froment and Bichoff [54]. In general, for high Peclet numbers (small deviations from plug-flow) the solution is not significantly affected by the type of boundary conditions.

Despite the renewed interest in the use of tubular reactors for EP [55, 56], the literature is still scarce in modeling studies and particularly in population balance models. Paquet and Ray [51, 52] did a good review on the use of this type of reactor for EP and laid the bases for their dynamic modeling. However, the model they proposed does not account for PSD, since it is based on the monodispersed approximation. To the best of our knowledge, Abad et al. [45] were the only ones who have attempted to model PSD using the tubular reactor formalism. The authors determined the RTD of their continuous loop reactor and found that it could be represented by a loop composed of two axial dispersion sections: the tube and the pump. They applied the set of equations described above, neglecting particle coagulation, to each of these sections and solved for the outlet PSD. The time evolution of the PSD predicted by the model agreed reasonably with the experimental distributions.

\subsubsection{Non-ideal stirred tank reactors}

Thus far, when dealing with STRs (independently of the operating mode), we have always assumed ideal behavior (i.e. homogeneity of the reactor contents). This is usually a reasonable approximation for small reactors, since efficient mixing can easily 
be achieved. However, this does not generally hold true for large-scale reactors, especially when operating at high solid contents. The PSD will be affected by such inhomogeneities, as the rates of nucleation, growth, and coagulation may vary from one zone of the reactor to another. For example, in a semi-batch process, the PSD may broaden as a result of the spatial gradients of monomer, initiator and surfactant.

In principle, the PSD in a non-ideal STR could be computed by solving the appropriate PBE (to be derived from Eq. (1)) together with the equations of continuity for each chemical species, and the Navier-Stokes equations. Nevertheless, such approach would require prohibitive computational times, since the solution of a population balance model based on the equations presented in Section 2.2 is already lengthy. In some research fields, like in precipitation modeling, this problem is being surmounted by using the method of moments to compute just the first moments of the distribution, instead of the full PSD (e.g. [57, 58]). Unfortunately, the method of moments is best suited for unimodal distributions; thus, this strategy would not be applicable to the situations where PSD models are most needed in EP applications.

As an alternative to the direct application of computational fluid dynamics (CFD), hybrid approaches have been proposed [59]. In those, CFD is used to determine the flow field, while the remaining (material and/or population) balances are subsequently solved in a custom-built compartmental network. Each compartment is assumed homogeneous and modeled as a CSTR, with inter-compartmental flow rates previously determined by CFD. The most delicate part of this method is probably the definition of the compartments, though some heuristic rules have been suggested [59].

This kind of approach has been used by Alopaeus et al. [60, 61] for the modeling of drop size distribution in liquid-liquid systems, and by Kiparissides' workgroup [62, 63] for the modeling of PSD in suspension polymerization. The PBEs governing these two systems are quite similar in the sense that they are both controlled by the rates of drop breakage and aggregation, which in turn depend on the rate of energy dissipation, $\varepsilon$. Hence, for these systems, $\varepsilon$ can be used as the main criterion for the definition of the compartmental network. This is most clearly seen in the models developed Kiparissides and co-workers, where the reactor was divided into just two compartments: impeller zone (high $\varepsilon$ ) and circulation zone (low $\varepsilon$ ).

In emulsion polymerization, however, the phenomena that control PSD (nucleation, growth and coagulation) depend to a much greater extent on the concentrations of the reactants (initiator, surfactant and monomer). The reader is 
referred to Section 3.1 for details on the relative importance of physicochemical factors and shear rate on the coagulation rate. Consequently, when modeling non-ideal EP reactors, the concentrations of the reactants (affected by the location of the feeding tubes, etc.) must play a key role in the definition of the compartments [59, 64]. This significantly complicates the situation with respect to the cases exposed in the previous paragraph. Rigopoulos and Jones [59] proposed to address this type of problems by means of a virtual tracer experiment that emulates the actual feeding policy.

Recently, Alexopoulos and Kiparissides [65] extended their two-compartment model to describe a semi-batch EP reactor. However, it is not clear how the authors defined the compartments. Defining the compartments based on $\varepsilon$, as they did for suspension polymerization, does not seem appropriate given the reasons presented above. Using the volumes of the compartments and the exchange flow rates as fitting parameters does not add much insight into system behavior in so far as predicting the impact of changing mixing conditions, viscosity, etc. are concerned. On the other hand, the approach did allow the authors to at least model a given reactor configuration - a point not devoid of interest in industrial conditions. 


\section{Coagulation modeling}

It is nowadays widely accepted that precursor particles have insufficient colloidal stability and therefore undergo limited coagulation, both when surfactant concentrations are below and above the CMC $[15,66]$. Despite this fact, coagulation is still frequently neglected in models developed for emulsion polymerization (see Table 1), perhaps because of the increased mathematical complexity and consequent solution time required to include it. However, the truth is that with actual computer power and numerical methods (see Section 4) this can no longer be considered a major obstacle to the inclusion of coagulation, even though population balance models accounting for this phenomenon are yet cumbersome to solve. As we will see here, nowadays, the problem is far more likely to be the quality of the models available to describe particle coagulation. The reader is referred to references [67-69] for an introduction to the stability of polymer colloids.

\subsection{Coagulation mechanisms}

Two distinct mechanisms may lead to particle coagulation in emulsion polymerization: perikinetic aggregation (due to Brownian motion), and shear or orthokinetic aggregation (due to transport by fluid motion). The contributions of these two mechanisms to the overall coagulation rate are not necessarily additive [70] and depend both on the polymerization recipe and the mixing conditions, namely, on particle size, shear rate and colloidal stability.

A useful rule of thumb can be established by computing the quotient of the rate coefficients derived for the limiting cases of pure perikinetic and orthokinetic aggregation of two non-interacting particles [71, 72]. It results that orthokinetic aggregation is favored by high shear rates and large particle sizes. Based on this formula and assuming what the authors claimed is a typical value for the average shear rate in a stirred vessel $\left(100 \mathrm{~s}^{-1}\right)$, Verduyn et al. [72] computed the critical particle diameter (at which both mechanisms are equally important) to be $0.5 \mu \mathrm{m}$. Ottewill [67] indicates that orthokinetic aggregation can become the dominant effect above $1 \mu \mathrm{m}$. From this range of particle diameters $(0.5-1.0 \mu \mathrm{m})$, a rough estimate of the relative importance of 
Brownian and shear aggregation can be obtained, but one should keep in mind that this is a very simplified picture of the reality. In the next paragraph, a more detailed treatment is presented.

In real emulsion systems, particles are subject to interaction forces and tend to collide as a result of both Brownian and bulk fluid motion. Melis et al. [70] theoretically analyzed such cases by numerically solving the pair probability equation for a system of two equal-sized particles subject to interaction forces of the DLVO type (see Section 3.2.1) in an axisymmetrical flow field. The authors found that for unstable systems (fast aggregation) the contributions from both mechanisms are independent, making it possible to compute the total aggregation rate by adding the two fluxes. In contrast, for more stable systems (slow aggregation) the two mechanisms are nonlinearly coupled, invalidating the flux additivity assumption. Based on their simulation results, the authors also proposed a criterion in terms of a modified Peclet number as a means to check the influence of fluid dynamics on the aggregation rate. This criterion clearly shows that, in addition to the particle diameter, the ionic strength of the medium also plays a role in determining the importance of fluid motion.

Some experimental studies have been conducted with the purpose of quantifying the importance of perikinetic and orthokinetic aggregation. Chern et al. [73] investigated the effect of several reaction variables on the stability of methyl methacrylate/butyl acrylate latexes. They found that the agitation speed does not significantly affect the coagulation process. Kemmere et al. [71, 74] studied the influence of process conditions on the coagulation behavior of polystyrene and polyvinyl acetate latexes. In a first study [71], concerning latexes with $25 \%$ solids content, coagulation was found to be governed by physicochemical factors, without any significant effect of energy dissipation rate, tank scale, impeller type or impeller diameter. Perikinetic aggregation was pointed to be the dominating mechanism for particle coagulation. In a subsequent work [74], the authors analyzed the influence of solids content on the colloidal stability of the abovementioned latex systems. They concluded that even for high-solids latexes (up to 50\%), perikinetic coagulation dominates over orthokinetic coagulation. The mixing conditions did not affect the colloidal stability of the latexes, although for polystyrene latexes they appeared to influence the extent of coagulum formation. Zubitur and Asúa [75, 76] analyzed the effect of the agitation rate on the emulsion copolymerization of styrene and butyl 
acrylate. The degree of agitation was found to affect the rate of mass transfer and the amount of coagulum formed, but no proof of orthokinetic coagulation was observed.

For modeling purposes, it is of paramount importance to determine the magnitude of orthokinetic aggregation with respect to Brownian agregation. This is because the case of pure Brownian aggregation is much simpler to treat than the combined mechanism case. In fact, accounting for both coagulation mechanisms in a rigorous manner, like Melis et al. [70] did, demands a very complex mathematical treatment. The computation of the total coagulation rate coefficient requires the numerical solution a second order partial differential equation, which may become prohibitive considering that such a problem must be solved simultaneously with the population balance equation. Fortunately, as shown in the last paragraph, there is experimental evidence that, for certain emulsion systems and operating conditions, Brownian aggregation is the only relevant mechanism. Of course, despite this general tendency, one should always examine the significance of orthokinetic aggregation for the system under consideration, either experimentally (most recommended) or theoretically.

For these reasons, as will be seen in the next section, only perikinetic aggregation is considered in the coagulation models developed by most authors working in emulsion polymerization. An exception to this rule, is the work of Forcolin et al. [77] where the authors employed the more complete approach developed by Melis et al. [70]. However, Forcolin et al. found a limited contribution of the fluid flow to the coagulation rate, thereby justifying this simplification.

As a final remark, note that if coagulum formation is significant, some means must be employed to take it into account. Unfortunately, this is not an easy task since coagulum may be formed by different mechanisms [78], and the available models are essentially semi-empirical [79-82].

\subsection{Modeling coagulation rate coefficients}

In general, perikinetic coagulation is the only mechanism of aggregation taken into account in the population balance models developed for EP, probably for the reasons cited in the previous section. Essentially, two distinct strategies have been used to estimate the rate of Brownian coagulation in EP: the DLVO theory of colloid 
stability, and semi-empirical approaches. Both of these methods are reviewed in the following sections.

\subsubsection{DLVO-based models}

A number of models based on the (classical and extended) DLVO theory [83, 84] have been proposed to predict the coagulation rate coefficients of latex particles stabilized both by anionic surfactants (Coen et al. [8, 22], Fortuny et al. [85], Kiparissides et al. [86], and Melis et al. [87, 88]), or of non-ionic surfactants (Immanuel et al. [13], Kammona et al. [48], and Lazaridis [89]). Next, we will take a look at the key steps of the DLVO approach, followed by a discussion of its major limitations. Finally, some modeling results are presented in order to evaluate the potentialities of this method.

\subsubsection{Overview of the DLVO approach}

The coagulation rate coefficient, $\beta$, between particles of swollen radii $r_{\mathrm{s} i}$ and $r_{\mathrm{s} j}$ is computed from the Fuchs' modification of the Smoluchowski equation for perikinetic aggregation [90, 91],

$$
\beta\left(r_{\mathrm{s} i}, r_{\mathrm{s} j}\right)=\frac{2 k_{\mathrm{B}} T}{3 \mu W\left(r_{\mathrm{s} i}, r_{\mathrm{s} j}\right)}\left(2+\frac{r_{\mathrm{s} i}}{r_{\mathrm{s} j}}+\frac{r_{\mathrm{s} j}}{r_{\mathrm{s} i}}\right)
$$

where $\mu$ is the viscosity of the continuous phase and $W$ is the Fuchs' stability ratio. The stability ratio is the inverse of the collision efficiency and accounts for the presence of colloidal and hydrodynamic interactions between the particles. An expression considering these two effects was given by Spielman [92],

$$
W=\left(r_{\mathrm{s} i}+r_{\mathrm{s} j}\right) \int_{r_{\mathrm{si}}+r_{\mathrm{s} j}}^{\infty} \frac{\exp \left(V_{\mathrm{T}} / k_{\mathrm{B}} T\right)}{G\left(R,\left(r_{\mathrm{s} i} / r_{\mathrm{s} j}\right)\right) R^{2}} d R
$$


where $R$ is the center-to-center distance between particles, $V_{\mathrm{T}}$ is the total particle interaction energy, and $G$ is the hydrodynamic interaction function which can be calculated with help from references [92-95]. This equation was used in references [13, $48,85,87-89]$ assuming $G=1$, i.e. neglecting the hydrodynamic interaction. However, these authors did not comment on the importance of this approximation, which only becomes valid when $R$ is large compared to the radii of the particles. Coen et al. [8] used a simpler expression to compute $W$ that only requires determining the maximum of $V_{\mathrm{T}}(R)$, but as remarked by Lazaridis et al. [89] such treatment may lead to large errors. Note that care must be taken while numerically evaluating the improper integral in Eq. (36) so that convergence is attained.

The total particle interaction energy, $V_{\mathrm{T}}$, is conventionally assumed to be the sum of all the attractive and repulsive (electrostatic and steric) contributions. This hypothesis has however been questioned by Einarson and Berg [96] and Porcel at al. [97], who argue that the electrostatic and steric potentials are not totally independent.

The van der Waals attractive potential energy is generally computed by the Hamaker-De Boer equation for two unlike spheres, retardation effects being neglected (e.g. [98]). The only parameter in this equation is the Hamaker constant, which accounts for the nature of the polymer particles (eventually different) and the interacting medium. Values for a number of polymers are given by Morrison and Ross [99].

The electrostatic repulsion potential energy, $V_{\mathrm{R}}$, for unlike particles is rather difficult to compute because no exact analytical solution exists and the different approximations available [100] are not valid for the entire range of radius, surface potential, and ionic strength typical of EP systems. Therefore, different choices have been made by researchers, but not always justifiable. For example, Coen et al. [8], Fortuny et al. [85], and Melis et al. [87, 88] used the Hogg-Healy-Fürstenau (HHF) expression, valid for constant surface potential, $\kappa r_{\mathrm{s} i}>>10$ and $\psi_{\mathrm{d}}<25 \mathrm{mV}$ [100-102]. Nevertheless, the condition imposed on the dimensionless particle size shows that the applicability of the HHF equation to the small precursor particles is questionable. Lazaridis et al. [89], on the other hand, used a set of three expressions to compute $V_{\mathrm{R}}$ according to the particle size. However, a similar approach had been used some years before by Feeney et al. [103], who concluded that these expressions significantly overestimate the repulsive barrier for the coagulation of unlike spheres. The absence of a reliable analytical expression for $V_{\mathrm{R}}$ raises a problem, since $V_{\mathrm{R}}$ has a strong influence 
on the values of stability ratio. If necessary, this difficulty could be overcome by using the exact non-linear treatment by Overbeek [100], but given the inherent limitations of the DLVO theory (see the next section) such treatment in not likely to worth the effort.

The number of EP models incorporating purely steric stabilization is still restricted. Lazaridis et al. [89] developed expressions for the steric potential energy between unlike particles stabilized by oligomeric non-ionic surfactants. The volume restriction and osmotic contributions were accounted for, and the extent of lateral migration controlled by means of an adjustable parameter. Immanuel et al. [13] described the steric repulsive potential due to non-ionic poly(ethylene oxide) surfactants through a simple formula given by Israelachvili [104], which accounts for osmotic and elastic contributions.

The particle surface coverage is a fundamental parameter for the computation of the repulsive potentials. Chemical equilibrium is invariably assumed between the aqueous phase and the surface of the particles. Some authors use a surfactant adsorption isotherm [105], but often the amount of free surfactant in the aqueous phase is neglected. The adsorption of ionic and non-ionic surfactants on latex particles is frequently well described by a simple Langmuir isotherm [106-108]. On the other hand, the adsorption of mixed ionic/non-ionic systems appears to be more complex to describe quantitatively due to the complex mechanisms involved [97, 109].

The last step in the computation of the electrostatic repulsive potential is to relate the diffuse potential, $\psi_{\mathrm{d}}$, to the total surface charge density of the particles, $\sigma_{0}$, given by the sum of the surface charges due to adsorbed surfactant, ionic end groups and in situ generated surfactant [15]. A number of authors [85, 86, 89] followed the approach given by Coen et al. [8] and used the Debye-Huckel or the Gouy-Chapman equations to relate $\sigma_{0}$ to the surface potential $\psi_{0}$, and then a Stern layer model to compute $\psi_{\mathrm{d}}$ from $\psi_{0}$. However, as noted by Melis et al. [88], such a procedure seems to be incorrect since the Debye-Huckel and Gouy-Chapman equations cannot be used to relate $\sigma_{0}$ to the surface potential $\psi_{0}$. An alternative formulation that overcomes this inconsistency was given by the same authors and recently extended by Sefcik et al. [110] to describe the charging of latexes stabilized by sodium dodecyl sulfate. 


\subsubsection{Limitations of the DLVO approach}

The use of the DLVO theory to compute the coagulation rate coefficients is becoming standard practice among researchers, and is sometimes presented as a guarantee of the quality of the model. Nevertheless, there is substantial evidence of the quantitative limitations of this theory with respect to the prediction of the aggregation rate of colloids [101, 111-113]. According to Behrens et al. [111], there are two major incoherencies: i) the sensitivity of aggregation rates to variations in ionic strength is overestimated; ii) the effect of particle size on the double layer repulsion has not been confirmed by aggregation experiments. These authors measured the influence of $\mathrm{pH}$ and ionic strength on the stability ratio of polystyrene carboxylated particles and observed that the DLVO theory only worked for low surface charges $\left(\sigma_{0}<3 \mathrm{mC} / \mathrm{m}^{2}\right)$ and low ionic strengths $(I<10 \mathrm{mM})$. A prior study [114] performed with polystyrene particles stabilized by sulfate groups led to the same conclusions. Behrens et al. [111] claim that the theory breaks down above these limits because the potential barrier is located at surface distances below 1-2 nm, where non-DLVO forces [104] are known to exist. Thus, simplified models not accounting for the structure of the solution and the particle surface (roughness, discrete nature of charges, etc.) cannot be expected to work in that region. These findings raise serious questions on the applicability of the DLVO theory to emulsion polymerization, since typical operating conditions would make the zone of 1-2 $\mathrm{nm}$ around the particle surface important for the calculation of the stability ratio. Besides, the usual picture of a precursor particle [15] is far from homogeneous in terms of geometry and surface charge density.

Another important aspect has to do with the fact that the DLVO theory supposes dilute dispersions, for which the influence of the surrounding particles on the pair of interacting particles can be neglected. In concentrated systems, however, the surrounding particles cause an effective reduction of the total potential energy of the interacting particles, and hence a lower stability ratio [115-117]. This may have important implications in the modeling of particle coagulation in emulsion polymerization, particularly for high solid content processes. In $a b$ initio polymerizations, particle formation often takes place at low solid contents, and thus one may claim that the effect of particle concentration on the stability ratio is perhaps not significant (although the remarks in the preceding paragraph still apply). On the 
contrary, the production of bimodal HSC latexes without intermediate seeds involves the in situ nucleation of a second population of particles in a latex of at least $50 \%(\mathrm{v} / \mathrm{v})$ polymer content. Under such circumstances, it is entirely conceivable that the concentration effect will be relevant.

A further aspect is the assumption that every latex particle in the system has the same (equilibrium) surfactant coverage (see the previous section). As perhaps first noted by Hansen and Ugelstad [118], this may not be true for the precursor particles formed by homogeneous nucleation, which may undergo coagulation at a faster rate than the rate of surfactant diffusion. This issue was also addressed by Giannetti [17], who compared the characteristic times for adsorption and coagulation, concluding that the surface coverage of the precursor particles cannot be evaluated from an adsorption isotherm. Obviously, this is not a limitation of the DLVO theory itself, but a consequence of applying an equilibrium hypothesis to a dynamic process such as emulsion polymerization.

The previous paragraphs clearly show that the DLVO approach is faced with two fundamental problems. First, the difficulty in obtaining reliable estimates for a number of input parameters, namely: the surface charge density, the diffuse potential and the electrostatic repulsion potential energy. It is worth noting that a deviation of $10 \%$ in $\sigma_{0}$ or $\psi_{\mathrm{d}}$ can lead to differences of two orders of magnitude in the predicted stability ratio. Second, and more important, the fact that the DLVO theory itself is possibly not applicable under emulsion polymerization conditions.

\subsubsection{Experimental validation}

Despite these objections, the DLVO theory remains essentially the only description of particle coagulation available when modeling the PSD in EP. In addition, it appears that by appropriately fitting certain model parameters, e.g. the Hamaker constant, it is possible to predict values of the coagulation rate coefficients in such a way that the model can be used to simulate experimental results. We will review some examples of this.

Coen et al. [8] were the first to develop a full PBM accounting for particle coagulation by means of the DLVO theory. The authors validated their model against 
experimental data on the ab initio and seeded polymerization of styrene. With respect to $a b$ initio systems, the model could adequately reproduce the effect of the surfactant (SDS) concentration on the final particle number, despite some mismatch around the CMC. The effect of electrolyte $(\mathrm{NaCl})$ addition on the particle number was well described by the model for surfactant concentrations above the CMC, but not below. At equal conversions, the general shape of the PSD was correctly predicted, but since the PSDs were essentially symmetrical this does not tell much about the model capabilities. Concerning the seeded experiments, the influence of the number and size of the seed particles on the amount of secondary nucleation (given by the ratio new/old particles) was successfully predicted by the model. However, a prior model by the same group [119] but without considering particle coagulation had also been capable of explaining the observed results.

Kiparissides et al. [86] developed a model to predict the effect of oxygen concentration on the emulsifier-free polymerization of vinyl chloride. Particle stability arises from the surface charge density generated by the sulfate groups, which is quantified by an empirical formula. The model performed well when validated against experimental data on conversion and average particle diameter obtained from a batch reactor operated at various initial oxygen and initiator concentrations. The U-shaped behavior of the average particle diameter with respect to the initial oxygen concentration was correctly explained by the model. This effect was attributed to the competition between the increase in radical production and the decrease in particle stability caused by the formation of $\mathrm{HCl}$. No comparison between experimental and computed PSDs was given.

Lazaridis et al. [89] compared the predictions of their steric stabilization model with experimental data on the copolymerization of vinyl acetate and butyl acrylate. The model was able to describe the effect of the surfactant structure (expressed by the number of stabilizing and adsorbing segments) on the cumulative conversion and the average particle size. For each type of oligomeric surfactant, the maximum surface coverage of the particles was used as a fitting parameter. The differences between experimental and predicted PSDs led the authors to conclude that the stabilization model underestimates heterocoagulation.

Immanuel et al. [13] developed a PBM for the emulsion copolymerization of vinyl acetate and butyl acrylate stabilized by a non-ionic poly(ethylene oxide) surfactant. Simulations were compared with experimental values of the solids content, 
number of particles and PSD, obtained for different feed profiles of the monomers, surfactant and initiator. The model showed poor quantitative validity regarding the evolution of the number of particles and PSD, despite the qualitative agreement.

It seems hard, if not impossible, to draw firm conclusions regarding the quality of the coagulation models used by these authors on the basis of the results reported. Essentially, this has to do with the fact that the coagulation models are not tested per se, but are used as a part of complete EP models that also account for particle nucleation and growth. As a result, errors may add, cancel out, or be irrelevant. The consequences are most evident when modeling ab initio polymerizations, since such systems can be described almost completely by conversion and number of particles. Thus, as long as the mismatches in nucleation and coagulation compensate for each other, the model will lead to the correct number of particles. Actually, one should keep in mind that level-one models (see Section 1), which do not account for particle coagulation, are also capable of the predicting reasonably well the evolution of conversion and number of particles for various systems (e.g. [6]). For systems with electrostatic stabilization, it is possible to overcome this cancellation effect by analyzing the influence of electrolyte addition, as did Coen et al. [8], since this only interferes with coagulation. Unfortunately, this test is not very practical because it requires data outside typical reaction conditions. Another alternative is to test the complete models under complex situations, as done by Immanuel et al. [13], since then one assures that agreement between simulation and experimental results will only be achieved if every piece of the model is working correctly. But again, in case of failure, it is difficult to identify the source(s) of error.

In the first instance, the obvious way to go around this interdependency problem would be to test the coagulation model under non-reacting conditions, as done by Melis et al. [88] and Fortuny et al. [85]. For example, the model could be compared with experimental data for the coagulation of monomer swollen particles or with experimental values of the stability ratio (obtained by turbidimetry, dynamic light scattering, etc. [120]). However, because of the shortcomings of the DLVO theory, this does not guarantee that the model will be successful in simulating coagulation under reaction conditions. In fact, these tests are carried out with latex particles having diameters necessarily higher that those of the particles for which coagulation may be significant in normal EP conditions. Moreover, the ionic strengths required to coagulate these particles are also above the typical values encountered in EP. Hence, even if the model is fitted for these conditions, the quantitative failure of the theory with respect to 
the effects of particle size and ionic strength will most likely lead to incorrect results when applied to predict the rate of coagulation in emulsion polymerization.

\subsubsection{Non DLVO-based models}

Some authors have preferred simpler empirical or semi-empirical approaches to the more fundamental (but not necessarily more accurate) DLVO theory. Unzueta and Forcada [121] developed a model to predict the effect of mixed anionic/non-ionic surfactant systems on the emulsion copolymerization of methyl methacrylate and butyl acrylate. The mixture of surfactants is described by a thermodynamic model for nonideal mixtures, from which the CMC of the surfactant mixture and the composition of the micelles are determined. The surface coverage of the latex particles is then computed from a Langmuir adsorption isotherm. Finally, the coagulation rate coefficients are defined, in analogy with Eq. (3.1), by a simple size-independent kernel of the type $\beta=\beta_{0} / W$. The stability ratio is an empirical function of the surface coverage of the particles. Despite the simplicity of the treatment, the results obtained with this semi-empirical model agreed well with experimental data concerning the effect of surfactant concentration and surfactant mixture composition on the evolution of conversion, average particle diameter, and copolymer composition. Furthermore, this is, to our knowledge, the only PBM dealing with mixed surfactant systems.

Another example of the use of simple coagulation models was given by Araújo et al. [12]. The authors defined a critical stability diameter and used it to divide the latex particles in two distinct populations. Particles with diameters below and above the critical value were designated, respectively, precursor and stable particles. Precursor particles can coagulate both with themselves (rate coefficient $\beta_{\mathrm{pp}}$ ) and with stable particles (rate coefficient $\beta_{\mathrm{ps}}$ ). Stable particles do not coagulate among themselves. Such a procedure may be seen as a discretization of the continuous kernel $\beta\left(r_{\mathrm{s} i}, r_{\mathrm{s} j}\right)$. The coagulation rate constants $\beta_{\mathrm{pp}}$ and $\beta_{\mathrm{ps}}$ were obtained by fitting the reactor model to the experimental data. The authors were able to describe, at least qualitatively, the main dynamic features of the emulsion copolymerization of vinyl acetate and Veova 10 in a continuous loop reactor. 


\section{Numerical solution of the population balance model}

\subsection{Introduction}

A large number of articles were published in the recent years dealing with the solution of population balances. A fairly complete review of the available numerical methods can be found in references [122-124]. Rather than aiming at an exhaustive description of all these works, this section will focus on methods that have proven their capacity to solve the type of PBEs involved in EP models. However, before looking at the resolution methods, it is useful to consider some of the difficulties faced when resolving equations of this form.

The PBEs described in this review may be seen as hyperbolic balance laws with special source terms [7, 125]. From the standpoint of numerical solution, two major difficulties are associated this type of partial differential equations (PDEs): i) the hyperbolic nature of PDE leads to numerical dispersion (i.e. nonphysical oscillations) and/or numerical diffusion (i.e. smearing) problems, which can only be surmounted by the use of sophisticated discretization methods [125]; ii) the evaluation of the net source function is a delicate matter, since any errors in the computation of the coagulation integrals (particularly in $\mathfrak{R}_{\text {coag }}^{+}$) will result in non-conservation of polymer mass [126].

The numerical solution of the PBEs is typically done in terms of the unswollen radius (see Section 2.1). The infinite radius domain must be truncated to a maximum value, $r_{\max }$, which has to be chosen wisely in order to control the finite-domain error [127, 128]. Some researchers have applied a logarithmic transformation to the particle size domain (e.g. [86, 127]). This kind of transformation improves the description of nucleation, since it expands the small particle region, but it tends to perform poorly as particles grow by polymerization and coagulation [128]. We thus recommend the use of uniform grids in terms of the unswollen radius.

The solution of PBE by the method of lines leads to a large set (set A) of ordinary differential equations (ODEs) in time that must be integrated together with the remaining ODEs comprising the reactor model (set B). Normally, the latter equations describe the evolution of the concentrations of initiator, surfactant, ion-oligoradicals, monomers, etc. In certain cases, e.g. when the concentration of ion-oligoradicals is computed from the corresponding transient balances, the total set of ODEs $(A+B)$ is 
highly stiff. This results in a waste of computational resources, because small time steps must be used for the solution of the complete system of ODEs. Immanuel et al. [123] proposed to overcome this problem by using a two-tier hierarchical solution strategy. In the first tier, the set B is solved, holding the PSD fixed. In the subsequent tier, the PSD (set A) is updated using the values computed from set B. The computation then proceeds to the next time step.

The calculation of the coagulation rate coefficients deserves a final remark. If Eqs. (35) and (36) are used for this purpose, the evaluation of the 2-dimensional function $\beta\left(v, v^{\prime}\right)$ will involve a considerable computational load, because of the integration process. Accordingly, if this procedure is repeated at each integration step, the model solution will be very time consuming. A good way to avoid this problem is to monitor the evolution of the parameters affecting the coagulation rate coefficients $\left(\sigma_{0}\right.$, $I, T$, etc. ) and to recompute $\beta\left(v, v^{\prime}\right)$ only when one of these parameters changes by more than a certain amount.

\subsection{Finite elements}

The finite element (FE) method $[129,130]$ is widely used in science and engineering for solving PDEs, and was first applied to PBEs by Gelbard and Seinfeld [127]. The technique of orthogonal collocation on finite elements (OCFE), in particular, has been used by several authors to describe the evolution of PSD in EP reactors (see Table 1). By means of the OCFE method, the PBE is semi-discretized and transformed into a system of differential algebraic equations (DAEs), whose unknowns are the values of the number density function at the collocation points. The reader is referred to references [30,47, 124, 130] for details on the implementation of this numerical technique. As shown in Table 1, the OCFE method has mostly been used in coagulation-free models. Araújo et al. [12] are apparently an exception to this rule, but recall that these authors used a simplified coagulation kernel. For the solution of coagulation-inclusive models, other discretization methods have been preferred, namely discretized population balances (DPBs) and finite difference/volume methods (see next section). 
In a recent theoretical study, Alexopoulos et al. [124] evaluated the performance of both the OCFE method and the discretization method of Lister et al. [131] for processes undergoing combined growth and aggregation. The effect of different kernels over a wide range of dimensionless growth and aggregation times was analyzed. The OCFE method was found to be, in general, more accurate. Nevertheless, for growth dominated problems, the results obtained with the OCFE method presented nonnegligible instabilities and dissipation. The spurious oscillations affecting the numerical solutions near the moving fronts could be reduced by adding an artificial diffusion term (a common practice in hyperbolic PDEs [132]), but obviously that further increased the amount of numerical dissipation. This stabilization trick was also used by Saldívar and Ray [46] in conjunction with the OCFE method. Rigopoulos and Jones [126] proposed a different type of FE scheme featuring enhanced stability and conservation of moments with respect to other FE methods, while being easy to implement and fast. The scheme consists of collocation on linear elements with a first order backward approximation for the growth term. However, this method suffers from significant dissipation, as is usual with first order upwind schemes.

The oscillation and/or dissipation problems reported in the above paragraph are characteristic of FE schemes for hyperbolic problems [129]. Classical finite difference and finite volume schemes also suffer from this limitation, perhaps even more seriously. The latter methods, however, have the advantage of being simpler to implement and computationally less demanding. This probably explains why the FE method has not been employed in coagulation-inclusive models, inherently heavier. In the following section, an alternative approach is described which we believe is more efficient.

\subsection{Finite differences/volumes}

The finite difference (FD) method of is one of the most popular for solving PDEs. The finite volume (FV) method is closely related to the FD method, but is derived from the integral form of the differential equations, a starting point that is reflected in a number of advantages [125].

The limitations of linear FD schemes when applied to hyperbolic problems are well documented $[129,132]$. The use of second order central (C2) schemes to discretize the convective term invariably leads to spurious oscillations, even with fine mesh 
spacing. Stable solutions can be obtained if the first order backward (B1) difference approximation is employed. Unfortunately, this upwind formula is only first order accurate and leads to excessive numerical diffusion; therefore a very fine grid is required throughout the entire domain to obtain correct solutions. The same difficulties are encountered with the FV formulation when first order upwind and second order central schemes are used to compute the particle flux at the cell boundaries [125]. Despite their limitations, the above mentioned schemes have been used by various authors to solve PBEs (see Table 1). For example, the inconvenience of using schemes of type C2 can be clearly seen in the plots given by Coen et al. [8].

In order to obtain high order $(\geq 2)$ accuracy and oscillation-free solutions, high resolution methods must be employed [125]. In particular, semi-discrete schemes have been developed that can be used to convert the PDE into a system of ODEs in time, which are then integrated (method of lines). Such methods can be applied both to FD and FV formulations and are fairly simple to implement. This approach has been in use for some time in other research fields, but only recently was it applied to PBEs [122, 133, 134]. As demonstrated by these recent works, the combination of the FV method with a semi-discrete high resolution scheme is particularly suited for the solution of PBEs. This is because: i) the difficulties related to the hyperbolic nature of the PBE (dispersion and dissipation) are subdued by the use of a high resolution scheme; ii) the FV method ensures that the number of particles is conserved [125]; iii) point sources, such as particle nucleation (see Section 2.1), can be easily treated; iv) the FV formulation is fully compatible with the simple yet efficient discretization methods developed for particle aggregation, namely the well-reputed fixed pivot technique of Kumar and Ramkrishna [135]. For these reasons, this approach is, in our opinion, the best choice for solving the full PBEs appearing in EP models.

The key steps of this method are outlined below, taking Eq. (15) as an example. The PBEs described in Sections 2.2.2 and 2.4.1 can be solved in a similar way. As depicted in Fig. 2, the truncated radius domain is subdivided into $M$ cells $C_{i} \equiv\left[r_{i-1 / 2}, r_{i+1 / 2}\right]$, with center $r_{i}=\left(r_{i-1 / 2}+r_{i+1 / 2}\right) / 2$, and size $\Delta r_{i}$. The average value of the density function over $C_{i}$ at time $t$ is,

$$
\bar{F}_{i}(t)=\frac{1}{\Delta r_{i}} \int_{r_{i-1 / 2}}^{r_{i+1 / 2}} F(r, t) d r=\frac{N_{i}(t)}{\Delta r_{i}}
$$


where $N_{i}(t)$ is the number of particles per unit volume of aqueous phase in that cell. According to the FV method, we do not solve the PBE directly, but its integrated version. Integrating Eq. (15) over the $i$ th cell and dividing by $\Delta r_{i}$ gives:

$$
\begin{aligned}
\frac{1}{V_{\mathrm{w}}} \frac{d}{d t}\left(V_{\mathrm{w}}\right. & \left.\bar{F}_{i}(t)\right)=-\frac{1}{\Delta r_{i}}\left(\hat{\Phi}_{i+1 / 2}-\hat{\Phi}_{i-1 / 2}\right)+\frac{Q^{\mathrm{in}} \phi_{\mathrm{w}}^{\text {in }}}{V_{\mathrm{w}}} \bar{F}_{i}^{\text {in }}(t)-\frac{Q^{\text {out }} \phi_{\mathrm{w}}^{\text {out }}}{V_{\mathrm{w}}} \bar{F}_{i}(t)+\frac{\delta_{i, 1}}{\Delta r_{i}} R_{\mathrm{nuc}}(t) \\
& +\frac{1}{\Delta r_{i}} \sum_{v_{i-1} \leq\left(v_{j}+v_{k}\right)<v_{i+1}}^{j \geq k}\left(1-1 / 2 \delta_{j, k}\right) \eta \beta\left(v_{j}, v_{k} ; t\right) \bar{F}_{j}(t) \bar{F}_{k}(t) \Delta r_{j} \Delta r_{k} \\
& -\bar{F}_{i}(t) \sum_{k=1}^{M} \beta\left(v_{i}, v_{k} ; t\right) \bar{F}_{k}(t) \Delta r_{k}
\end{aligned}
$$

Here, $\hat{\Phi}_{i+1 / 2}$ stands for the numerical approximation of the flux $\Phi \equiv \hat{k}(r, t) F(r, t)$ at $r=r_{i+1 / 2}$. For instance, if a first order upwind scheme is used, the numerical flux reads $\hat{\Phi}_{i+1 / 2} \equiv \hat{k}\left(r_{i+1 / 2}, t\right) \bar{F}_{i}(t)$. In high resolution methods, the numerical flux is computed by a suitable high order non-linear scheme [125, 136, 137]. A good option is to employ the so-called weighted essentially non-oscillatory (WENO) methods [136, 138, 139], in particular the third and fifth order schemes. The corresponding algorithms are explained in detail by Shu [136]. Referring back to Eq. (38), note that the inclusion of nucleation is quite straightforward, the corresponding term being accounted for in the first cell. The last two terms on the RHS of Eq. (38) are simply the discretized equations for pure aggregation developed by Kumar and Ramkrishna [135]. Although other discretization schemes exist [131, 140-142], the fixed pivot technique is the suggested choice, since this method is robust, versatile, accurate, and simple to implement [141].

The approach just described is applicable to problems involving simultaneous nucleation, growth and coagulation. In addition, it is both oscillation-free and accurate, can be used with arbitrary non-uniform grids, and is easier to implement than the OCFE method. This method is also superior to the moving grid technique proposed by Kumar and Ramkrishna [143], as the latter is not well suited for problems involving nucleation. In order to illustrate the capacities of this approach, let us look at some simulation results. Fig. 3 depicts the growth of a $100 \mathrm{~nm}$-seed. The advantages of the fifth order WENO scheme over the first order upwind scheme are quite evident. Note that for 
$M=300$ the B1 solution still reveals significant numerical diffusion. In contrast, the two WENO5 curves are indistinguishable. Fig. 4 illustrates the synthesis of a bimodal latex starting from a $400 \mathrm{~nm}$-seed. In this simulation we employed a simple coagulation kernel like the one used by Araújo et al. [12]. Population balance models are particularly useful for this type of processes, as they allow one to describe the sizes and relative amounts of both populations. 


\section{Conclusions}

PBEs bring added dimension to the modeling of EP in so far as they provide a structure that can be used to link reaction kinetics, particle nucleation and growth, as well as coagulation to the PSD. This type of model should, in theory, allow one to develop significant insights into the relationship between the reactor, reaction conditions and latex quality (PSD, MWD, composition distribution, etc.) in ways that cannot be achieved using level-one models (i.e. assuming a monodisperse PSD).

The objective of this review was to evaluate the progress in EP modeling using PBEs. It is clear that significant advances have been made, but certain challenges subsist. As with many types of problems, the more complete the models, the more computationally intensive they become. Even if one sets aside difficulties related to modeling coagulation, it is clear that PBEs are difficult to solve if one includes full hydrodynamic and/or kinetic models. Therefore, while useful, a number of PBE-based approaches that one finds in the literature are obliged to rely upon assumptions about, among other things, the distribution of free radicals in the particles in order to reduce model complexity to a computationally manageable level. However, with the correct choice of numerical solution techniques, full PBEs, and eventually two dimension PBE models of EP will allow us to more fully describe the evolution of the PSD coupled with another property distribution such as the composition of different particles.

On the other hand, it appears that the major obstacle to a more widespread and effective use of PBEs in modeling the PSD is not a challenge intrinsic to the PBEs themselves, rather it is related to a lack of physical models of some of the more important phenomena in EP. Of course, a number of difficulties are encountered when including coagulation, not the least of which is the inherent uncertainty of modeling the particle coagulation rate using the only available tool - the DLVO theory. It is nevertheless true that a judicious choice of model parameters by certain authors have allowed them to successfully model EP using DLVO and either zero-one or PB kinetics. An obvious conclusion is that a better model of particle coagulation is needed if we are to increase the accuracy and robustness of PBEs in modeling the PSD of emulsion polymerization. Such a model should include: ortho- and perikinetic contributions to coagulation, a means of assessing the stability of particles on length scale commensurate with emulsion polymerization, as well as a means of including the influence of the composition of mixed surfactant systems. To be fair, it is entirely 
unclear from the literature reviewed here how one could go about doing that! In addition, similar difficulties concerning uncertainties in the modeling of EP kinetics have been succinctly cited by van Herk and German [144].

In conclusion, PBEs are a promising tool for the modeling of various important aspects of emulsion polymerization, not the least of which is the inclusion of the development and the contribution of complex PSDs. Very positive advances in the use of this type of model have been made over the course of the last decade, and reasonable approximations and simplifications of complex physical parameters have been used with success in a number of publications. Advances in inexpensive computational power and in the development of evermore sophisticated numerical solution algorithms, coupled with progress in the area of modeling emulsion stability will make PBEs ubiquitous tools for the modeling of EP in the near future.

\section{Acknowledgements}

H. M. Vale wishes to thank the Portuguese Science and Technology Foundation for financial support (grant SFRH/BD/10513/2002). The authors also wish to thank our numerous colleagues who partook of their time to discuss some of their work in the field of PBEs and EP. Any errors of interpretation are ours alone. 


\section{References}

[1] Guyot A, Chu F, Schneider M, Graillat C, McKenna TF. High solid content latexes. Prog. Polym. Sci. 2002;27:1573-1615.

[2] Penlidis A, MacGregor JF, Hamielec AE. Mathematical modeling of emulsion polymerization reactors: a population balance approach and its applications. In: Provder T. Computer Applications in the Polymer Laboratory. Washington DC:American Chemical Society, 1986. p. 219-240.

[3] Min KW, Ray WH. On the mathematical modeling of emulsion polymerization reactors. J. Macromol. Sci., Revs. Macromol. Chem. 1974;C11:177-255.

[4] Saldívar E, Dafniotis P, Ray WH. Mathematical modeling of emulsion copolymerization reactors. I. Model formulation and application to reactors operating with micellar nucleation. J. Macromol. Sci., Revs. Macromol. Chem. Phys. 1998;C38:207-325.

[5] Dubé MA, Soares JBP, Penlidis A, Hamielec AE. Mathematical modeling of multicomponent chain-growth polymerizations in batch, semibatch, and continuous reactors: a review. Ind. Eng. Chem. Res. 1997;36:966-1015.

[6] Gao J, Penlidis A. Mathematical modeling and computer simulator/database for emulsion polymerizations. Prog. Polym. Sci. 2002;27:403-535.

[7] Ramkrishna D. Population balances. Theory and applications to particulate systems in engineering. San Diego:Academic Press, 2000.

[8] Coen EM, Gilbert RG, Morrison BR, Leube H, Peach S. Modelling particle size distributions and secondary particle formation in emulsion polymerization. Polymer 1998;39:7099-7112.

[9] Rawlings JB, Ray WH. The modeling of batch and continuous emulsion polymerization reactors. Part I: model formulation and sensivity to parameters. Polym. Eng. Sci. 1988;28:237-256.

[10] Mahoney AW, Ramkrishna D. Efficient solution of population balance equations with discontinuities by finite elements. Chem. Eng. Sci. 2002;57:1107-1119.

[11] Hulburt HM, Katz S. Some problems in particle technology. Chem. Eng. Sci. 1964;19:555-574. 
[12] Araújo PH, de la Cal JC, Asúa JM, Pinto JC. Modeling particle size distribution (PSD) in emulsion copolymerization reactions in a continuous loop reactor. Macromol. Theory Simul. 2001;10:769-779.

[13] Immanuel CD, Doyle III FJ, Cordeiro CF, Sundaram SS. Population balance PSD model for emulsion polymerization with steric stabilizers. AIChE J. 2003;49:1392-1404.

[14] Lattuada M, Wu H, Sandkühler P, Sefcik J, Morbidelli M. Modelling of aggregation kinetics of colloidal systems and its validation by light scattering measurements. Chem. Eng. Sci. 2004;59:1783-1798.

[15] Gilbert RG. Emulsion polymerization. A mechanistic approach. San Diego:Academic Press, 1995.

[16] Min KW, Ray WH. The computer simulation of batch emulsion polymerization reactors through a detailed mathematical model. J. Appl. Polym. Sci. 1978;22:89-112.

[17] Giannetti E. Nucleation mechanisms and particle size distributions of polymer colloids. AIChE J. 1993;39:1210-1227.

[18] Giannetti E. Comprehensive theory of particle growth in the Smith-Ewart interval II of emulsion polymerization systems. Macromolecules 1990;23:47484759.

[19] Gilbert RG. Modelling rates, particle size distributions and molar mass distributions. In: Lovell PA, El-Aasser MS. Emulsion Polymerization and Emulsion Polymers. Chichester:John Wiley \& Sons, 1997. p. 164-203.

[20] Lichti G, Gilbert RG, Napper DH. The growth of polymer colloids. J. Polym. Sci., Polym. Chem. Ed. 1977;15:1957-1971.

[21] Coen EM, Gilbert RG. Particle size distributions. In: Asúa JM. Polymeric Dispersions: Principles and Applications. Dordrecht:Kluwer Academic Publishers, 1997. p. 67-78.

[22] Coen EM, Peach S, Morrison BR, Gilbert RG. First-principles calculation of particle formation in emulsion polymerization: pseudo-bulk systems. Polymer 2004;45:3595-3608.

[23] Asúa JM, Sudol ED, El-Aasser MS. Radical desorption in emulsion polymerization. J. Polym. Sci., Part A: Polym. Chem. 1989;27:3903-3913. 
[24] Casey BS, Morrison BR, Maxwell IA, Gilbert RG, Napper DH. Free radical exit in emulsion polymerization. I. Theoretical model. J. Polym. Sci., Part A: Polym. Chem. 1994;32:605-630.

[25] Asúa JM. A new model for radical desorption in emulsion polymerization. Macromolecules 2003;36:6245-6251.

[26] Gilbert RG Personal communication 22/06/04. 2004.

[27] Maeder S, Gilbert RG. Measurement of transfer constant for butyl acrylate freeradical polymerization. Macromolecules 1998;31:4410-4418.

[28] Prescott SW. Chain-length dependence in living/controlled free-radical polymerizations: physical manifestation and Monte Carlo simulation of reversible transfer agents. Macromolecules 2003;36:9608-9621.

[29] Zeaiter J, Romagnoli JA, Barton GW, Gomes VG, Hawkett BS, Gilbert RG. Operation of semi-batch emulsion polymerization reactors: modelling, validation and effect of operating conditions. Chem. Eng. Sci. 2002;57:29552969.

[30] Crowley TJ, Meadows ES, Kostoulas E, Doyle III FJ. Control of particle size distribution described by a population balance model of semibatch emulsion polymerization. J. Proc. Cont. 2000;10:419-432.

[31] Meadows ES, Crowley TJ, Immanuel CD, Doyle III FJ. Nonisothermal modeling and sensivity studies for batch and semibatch emulsion polymerization of styrene. Ind. Eng. Chem. Res. 2003;42:555-567.

[32] Poehlein GW. Continuous processes. In: Lovell PA, El-Aasser MS. Emulsion Polymerization and Emulsion Polymers. Chichester:John Wiley \& Sons, 1997. p. 277-292.

[33] Scheren PA, Russell GT, Sangster DF, Gilbert RG, German AL. Chain-lengthdependent termination rate processes in free-radical polymerizations. 3. Styrene polymerizations with and without added inert diluent as an experimental test of model. Macromolecules 1995;28:3637-3649.

[34] Buback M, Egorov M, Junkers T, Panchenko E. Free-radical termination kinetics studied using a novel SP-PLP-ESR technique. Macromol. Rap. Comm. 2004;25:1004-1009.

[35] Smith WV, Ewart RM. Kinetics of emulsion polymerization. J. Chem. Phys. 1948;16:592-599. 
[36] O'Toole JT. Kinetics of emulsion polymerization. J. Appl. Polym. Sci. 1965;9:1291-1297.

[37] Ugelstad J, Mork PC, Aasen JO. Kinetics of emulsion polymerization. J. Polym. Sci., Polym. Chem. Ed. 1967;5:2281-2288.

[38] Li B-G, Brooks BW. Prediction of the average number of radicals per particle for emulsion polymerization. J. Polym. Sci., Part A: Polym. Chem. 1993;31:2397-2402.

[39] Prescott SW, Ballard MJ, Gilbert RG. Average termination rate coefficients in emulsion polymerization: effect of compartmentalization on free-radical lifetimes. J. Polym. Sci., Part A: Polym. Chem. 2005;43:1076-1089.

[40] Russell GT, Gilbert RG, Napper DH. Chain-length-dependent termination rate processes in free-radical polymerizations. 1. Theory. Macromolecules 1992;25:2459-2469.

[41] Storti G, Carrà S, Morbidelli M, Vita G. Kinetics of multimonomer emulsion polymerization. The pseudo-homopolymerization approach. J. Appl. Polym. Sci. $1989 ; 37: 2443-2467$.

[42] Chen S-A, Wu K-W. Emulsion polymerization: theory of particle size distribution in copolymerization system. J. Polym. Sci., Part A: Polym. Chem. 1988;26:1487-1506.

[43] Storti G, Morbidelli M, Carrà S. Detailed modeling of multicomponent emulsion polymerization systems. In: Provder T. Computer applications in applied polymer science II. New York:ACS, 1989. p. 379-402.

[44] Abad C, de la Cal JC, Asúa JM. Emulsion copolymerization in continuous loop reactors. Chem. Eng. Sci. 1994;49:5025-5037.

[45] Abad C, de la Cal JC, Asúa JM. Modelling nucleation and particle growth in emulsion copolymerization in continuous loop reactors. Macromol. Symp. 1995;92:195-204.

[46] Saldívar E, Ray WH. Mathematical modeling of emulsion copolymerization reactors: experimental validation and application to complex systems. Ind. Eng. Chem. Res. 1997;36:1322-1336.

[47] Immanuel CD, Cordeiro CF, Sundaram SS, Meadows ES, Crowley TJ, Doyle III FJ. Modeling of particle size distribution in emulsion co-polymerization: comparison with experimental data and parametric sensivity studies. Comp. Chem. Eng. 2002;26:1133-1152. 
[48] Kammona O, Pladis P, Frantzikinakis CE, Kiparissides C. A comprehensive experimental and theoretical investigation of the styrene/2-ethylhexyl acrylate emulsion copolymerization. Macromol. Chem. Phys. 2003;204:983-999.

[49] Soares JBP, Hamielec AE. Transport phenomena in emulsion polymerization reactors. In: Asúa JM. Polymeric Dispersions: Principles and Applications. Dordrecht:Kluwer Academic Publishers, 1997. p. 289-304.

[50] Poehlein GW. Reaction engineering for emulsion polymerization. In: Asúa JM. Polymeric Dispersions: Principles and Applications. Dordrecht:Kluwer Academic Publishers, 1997. p. 305-331.

[51] Paquet DA, Ray WH. Tubular reactors for emulsion polymerization: I. Experimental investigation. AIChE J. 1994;40:73-87.

[52] Paquet DA, Ray WH. Tubular reactors for emulsion polymerization: II. Model comparisons with experiments. AIChE J. 1994;40:88-96.

[53] Abad C, de la Cal JC, Asúa JM. The loop process. In: Asúa JM. Polymeric Dispersions: Principles and Applications. Dordrecht:Kluwer Academic Publishers, 1997. p. 333-347.

[54] Froment GF, Bischoff KB. Chemical reactor analysis and design. New York:John Wiley \& Sons, 1990.

[55] Poormahdian S, Bataille P. Emulsion copolymerization in a tubular reactor. J. Appl. Polym. Sci. 2000;75:833-842.

[56] Ouzineb K, Graillat C, McKenna TF. Continuous tubular reactors for latex production: conventional emulsion and miniemulsion polymerizations. J. Appl. Polym. Sci. 2004;91:2195-2207.

[57] Wei H, Zhou W, Garside J. Computational fluid dynamics modeling of the precipitation process in a semibatch crystallizer. Ind. Eng. Chem. Res. 2001;40:5255-5261.

[58] Jaworski Z, Nienow AW. CFD modelling of continuous precipitation of barium sulphate in a stirred tank. Chem. Eng. J. 2003;91:167-174.

[59] Rigopoulos S, Jones A. A hybrid CFD-reaction engineering framework for multiphase reactor modelling: basic concept and application to bubble column reactors. Chem. Eng. Sci. 2003;58:3077-3089.

[60] Alopaeus V, Koskinen J, Keskinen KI. Simulation of the population balances for liquid-liquid systems in a nonideal stirred tank. Part 1 Description and qualitative validation of the model. Chem. Eng. Sci. 1999;54:5887-5899. 
[61] Alopaeus V, Koskinen J, Keskinen KI, Majander J. Simulation of the population balances for liquid-liquid systems in a nonideal stirred tank. Part 2-parameter fitting and the use of the multiblock model for dense dispersions. Chem. Eng. Sci. 2002;57:1815-1825.

[62] Maggioris D, Goulas A, Alexopoulos AH, Chatzi E, Kiparissides C. Prediction of particle size distribution in suspension polymerization reactors: effect of turbulence nonhomogeneity. Chem. Eng. Sci. 2000;55:4611-4627.

[63] Alexopoulos AH, Maggioris D, Kiparissides C. CFD analysis of turbulence nonhomogeneity in mixing vessels. A two-compartment model. Chem. Eng. Sci. 2002;57:1735-1752.

[64] Arevalillo A, Asúa JM. CFD applied to emusion polymerization reactors. In: McKenna TF, Bourgeat-Lami E, Elaissari A. International Symposium on Polymers in Dispersed Media. Colloids: from Preparation to Applications. Lyon, 2004. p. 172-173.

[65] Alexopoulos AH, Kiparissides C. Prediction of particle size distribution in nonhomogeneous particulate systems. Two compartment model of an emulsion polymerization reactor. In: McKenna TF, Bourgeat-Lami E, Elaissari A. International Symposium on Polymers in Dispersed Media. Colloids: from Preparation to Applications. Lyon, 2004. p. 34-36.

[66] Hansen FK, Ugelstad J. Particle nucleation in emulsion polymerization. I. A theory for homogeneous nucleation. J. Polym. Sci., Polym. Chem. Ed. 1978;16:1953-1979.

[67] Ottewill RH. Stability of polymer colloids. In: Asúa JM. Polymeric Dispersions: Principles and Applications. Dordrecht:Kluwer Academic Publishers, 1997. p. 31-48.

[68] Ottewill RH. Stabilization of polymer colloid dispersions. In: Lovell PA, ElAasser MS. Emulsion Polymerization and Emulsion Polymers. Chichester:John Wiley \& Sons, 1997. p. 37-58.

[69] Blackley DC. Polymer latices, science and technology. Fundamental principles. London:Chapman \& Hall, 1997.

[70] Melis S, Verduyn M, Storti G, Morbidelli M, Baldyga J. Effect of fluid motion on the aggregation of small particles subject to interaction forces. AIChE J. 1999;45:1383-1393. 
[71] Kemmere MF, Meuldijk J, Drinkenburg AA, German AL. Aspects of coagulation during emulsion polymerization of styrene and vinyl acetate. J. Appl. Polym. Sci. 1998;69:2409-2421.

[72] Verduyn M, Sefcik J, Storti G, Morbidelli M. Particle aggregation in polymer latexes. In: Daniels ES, Sudol ED, El-Aasser MS. Polymer Colloids.ACS, 2002. p. 23-39.

[73] Chern CS, Hsu H, Lin FY. Stability of acrylic latices in a semibatch reactor. J. Appl. Polym. Sci. 1996;60:1301-1311.

[74] Kemmere MF, Meuldijk J, Drinkenburg AA, German AL. Colloidal stability of high-solids polystyrene and polyvinyl acetate latices. J. Appl. Polym. Sci. 1999;74:1780-1791.

[75] Zubitur M, Asúa JM. Factors affecting kinetics and coagulum formation during the emulsion copolymerization of styrene/butyl acrylate. Polymer 2001;42:59795985.

[76] Zubitur M, Asúa JM. Agitation effects in the semicontinuous emulsion polymerization of styrene and butyl acrylate. J. Appl. Polym. Sci. 2001;80:841851.

[77] Forcolin S, Marconi AM, Ghielmi A, Butté A, Storti G, Morbidelli M. Coagulation phenomena in emulsion polymerization of vinyl chloride. Plastics, Rubber and Composites 1999;28:109-115.

[78] Vanderhoff JW. The formation of coagulum in emulsion polymerization. In: Bassett DR, Hamielec AE. Emulsion polymers and emulsion polymerization. Washington DC:ACS, 1981. p. 199-208.

[79] Lowry V, El-Aasser MS, Vanderhoff JW, Klein A. Mechanical coagulation in emulsion polymerizations. J. Appl. Polym. Sci. 1984;29:3925-3935.

[80] Matejícek A, Pivonková A, Kaška J, Ditl P, Formánek L. Influence of agitation on the creation of coagulum during the emulsion polymerization of the system styrene-butylacrylate-acrylic acid. J. Appl. Polym. Sci. 1988;35:583-591.

[81] Chern CS, Kuo YN. Shear-induced coagulation kinetics of semibatch seeded emulsion polymerization. Chem. Eng. Sci. 1996;51:1079-1087.

[82] Lowry V, El-Aasser MS, Vanderhoff JW, Klein A, Silebi CA. Kinetics of agitation-induced coagulation of high-solid latexes. J. Colloid Interface Sci. 1986;112:521-529. 
[83] Verwey EJW, Overbeek JTG. Theory of the stability of lyophobic colloids. Mineola:Dover Publications, 1999.

[84] Hidalgo-Álvarez R, Martín A, Fernández A, Bastos D, Martínez F, de las Nieves FJ. Electrokinetic properties, colloidal stability and aggregation kinetics of polymer colloids. Adv. Colloid Interface Sci. 1996;67:1-118.

[85] Fortuny M, Graillat C, McKenna TF. Coagulation of anionically stabilized polymer particles. Ind. Eng. Chem. Res. 2004;43:7210-7219.

[86] Kiparissides C, Achilias DS, Frantzikinakis CE. The effect of oxygen on the kinetics and particle size distribution in vinyl chloride emulsion polymerization. Ind. Eng. Chem. Res. 2002;41:3097-3109.

[87] Melis S, Ghielmi A, Storti G, Morbidelli M. Aggregation phenomena in emulsion polymerization of vinyl chloride. Entropie 1998;212/213:65-72.

[88] Melis S, Kemmere MF, Meuldijk J, Storti G, Morbidelli M. A model for the coagulation of polyvinyl acetate particles in emulsion. Chem. Eng. Sci. 2000;55:3101-3111.

[89] Lazaridis N, Alexopoulos AH, Chatzi E, Kiparissides C. Steric stabilization in emulsion polymerization using oligomeric nonionic surfactants. Chem. Eng. Sci. 1999;54:3251-3261.

[90] Smoluchowski M. Versuch einer mathematischen theorie der koagulationskinetik kolloider lösungen. Z. Phys. Chem. 1917;92:129.

[91] Fuchs N. Z. Phys. 1934;89:736-743.

[92] Spielman LA. Viscous interactions in Brownian coagulation. J. Colloid Interface Sci. 1970;33:562-571.

[93] Batchelor GK. Brownian diffusion of particles with hydrodynamic interaction. J. Fluid Mech. 1976;74:1-26.

[94] Honig EP, Roebersen GJ, Wiersema PH. Effect of hydrodynamic interaction on the coagulation rate of hydrophobic colloids. J. Colloid Interface Sci. 1971;36:97-109.

[95] Jeffrey DJ, Onishi Y. Calculation of the resistance and mobility functions for two unequal rigid spheres in low-Reynolds-number flow. J. Fluid Mech. 1984;139:261-290.

[96] Einarson MB, Berg JC. Electrosteric stabilization of colloidal latex dispersions. J. Colloid Interface Sci. 1993;155:165-172. 
[97] Porcel R, Jódar AB, Cabrerizo MA, Hidalgo-Álvarez R, Martín-Rodríguez A. Sequential adsorption of Triton X-100 and sodium dodecyl sulfate onto positively and negatively charged polystyrene latexes. J. Colloid Interface Sci. 2001;239:568-576.

[98] Lyklema J. Fundamentals of interface and colloid science. Vol I: Fundamentals. London:Academic Press, 1995.

[99] Morrison ID, Ross S. Colloidal dispersions. New York:Wiley, 2002.

[100] Kihira H, Matijevic E. An assessment of heterocoagulation theories. Adv. Colloid Interface Sci. 1992;42:1-31.

[101] Kihira H, Ryde N, Matijevic E. Kinetics of heterocoagulation 1. A comparison of theory and experiment. Colloids Surf. 1992;64:317-324.

[102] Hogg R, Healy TW, Fürstenau. Mutual coagulation of colloidal dispersions. Trans. Faraday Soc. 1966;62:1638-1651.

[103] Feeney PJ, Napper DH, Gilbert RG. Surfactant-free emulsion polymerizations: predictions of the coagulative nucleation theory. Macromolecules 1987;20:29222930.

[104] Israelachvili J. Intermolecular and surface forces. San Diego:Academic Press, 1992.

[105] Zhu B-Y, Gu T. Surfactant adsorption at solid-liquid interfaces. Adv. Colloid Interface Sci. 1991;37:1-32.

[106] Lin S-Y, Dong C, Hsu T-J, Hsu C-T. Determination of adsorption of an ionic surfactant on latex from surface tension measurements. Colloids and Surfaces A: Physicochem. Eng. Aspects 2002;196:189-198.

[107] Romero-Cano MS, Martín-Rodríguez A, Chauveteau G, de las Nieves FJ. Colloidal stabilization of polystyrene particles by adsorption on nonionic surfactants. I. Adsorption study. J. Colloid Interface Sci. 1998;198:266-272.

[108] Colombié D, Landfester K, Sudol ED, El-Aasser MS. Determination of the adsorption isotherm of the nonionic surfactant Triton X-405 on polystyrene latex particles using H NMR. J. Colloid Interface Sci. 1998;202:554-557.

[109] Colombié D, Landfester K, Sudol ED, El-Aasser MS. Competitive adsorption of the anionic surfactant SLS and the nonionic surfactant Triton X-405 on polystyrene latex particles. Langmuir 2000;16:7905-7913.

[110] Sefcik J, Verduyn M, Storti G, Morbidelli M. Charging of latex particles stabilized by sulfate surfactant. Langmuir 2003;19:4778-4783. 
[111] Behrens SH, Christl DI, Emmerzael R, Schurtenberger P, Borkovec M. Charging and aggregation properties of carboxyl latex particles: experiments versus DLVO theory. Langmuir 2000;16:2566-2575.

[112] Swanton SW. Modelling colloid transport in groundwater; The prediction of colloid stability and retention behavior. Adv. Colloid Interface Sci. 1995;54:129-208.

[113] Kihira H, Matijevic E. Kinetics of heterocoagulation. 3. Analysis of effects causing the discrepancy between the theory and experiment. Langmuir $1992 ; 8: 2855-2862$.

[114] Behrens SH, Semmler M, Borkovec M. Aggregation in sulfate latex suspensions: the role of charge for stability predictions. Prog. Colloid Polym. Sci. 1998;110:66-69.

[115] SenGupta AK, Papadopoulos KD. Stability of concentrated colloids: the controlling parameters. J. Colloid Interface Sci. 1998;203:345-353.

[116] Hsu J-P, Liu B-T. Critical coagulation concentration of a colloidal suspension at high particle concentrations. J. Phys. Chem. B 1998;102:334-337.

[117] Hütter M. Coagulation rates in concentrated colloidal suspensions studied by Brownian dynamics simulation. Phys. Chem. Chem. Phys. 1999;1:4429-4436.

[118] Hansen FK, Ugelstad J. Particle nucleation in emulsion polymerization. III. Nucleation in systems with anionic emulsifier investigated by seeded and unseeded polymerization. J. Polym. Sci., Polym. Chem. Ed. 1979;17:3047-3067.

[119] Morrison BR, Gilbert RG. Conditions for secondary particle formation in emulsion polymerization systems. Macromol. Symp. 1995;92:13-30.

[120] Holthoff H, Egelhaaf SU, Borkovec M, Schurtenberger P, Sticher H. Coagulation rate measurements of colloidal particles by simultaneous static and dynamic light scattering. Langmuir 1996;12:5541-5549.

[121] Unzueta E, Forcada J. Modeling the effect of mixed emulsifier systems in emulsion copolymerization. J. Appl. Polym. Sci. 1997;66:445-458.

[122] Attarakih MM, Bart H-J, Faqir NM. Numerical solution of the spatially distributed population balance equation describing the hydrodynamics of interacting liquid-liquid dispersions. Chem. Eng. Sci. 2004;59:2567-2592.

[123] Immanuel CD, Doyle III FJ. Computationally efficient solution of population balance models incorporating nucleation, growth and coagulation: application to emulsion polymerization. Chem. Eng. Sci. 2003;58:3681-3698. 
[124] Alexopoulos AH, Roussos AI, Kiparissides C. Part I: Dynamic evolution of the particle size distribution in particulate processes undergoing combined particle growth and aggregation. Chem. Eng. Sci. 2004;59:5751-5769.

[125] LeVeque RJ. Finite volume methods for hyperbolic problems. Cambridge:Cambridge University Press, 2002.

[126] Rigopoulos S, Jones A. Finite-element scheme for solution of the dynamic population balance equation. AIChE J. 2003;49:1127-1139.

[127] Gelbard F, Seinfeld JH. Numerical solution of the dynamic equation for particulate systems. J. Comput. Phys. 1978;28:357-375.

[128] Nicmanis M, Hounslow MJ. Finite-element methods for steady-state population balance equations. AIChE J. 1998;44:2258-2272.

[129] Lapidus L, Pinder GF. Numerical solution of partial differential equations in science and engineering. New York:John Wiely \& Sons, 1999.

[130] Finlayson BA. Nonlinear analysis in chemical engineering. New York:McGraw Hill, 1980.

[131] Litster JD, Smit DJ, Hounslow MJ. Adjustable discretized population balance for growth and aggregation. AIChE J. 1995;41:591-603.

[132] LeVeque RJ. Numerical methods for conservation laws. Basel:Birkhäuser, 1992.

[133] Lim YI, Le Lann JM, Meyer XM, Joulia X, Lee G, Yoon ES. On the solution of population balance equations (PBE) with accurate front tracking methods in practical crystallization processes. Chem. Eng. Sci. 2002;57:3715-3732.

[134] Motz S, Mitrovic A, Gilles E-D. Comparison of numerical methods for the simulation of dispersed phase systems. Chem. Eng. Sci. 2002;57:4329-4344.

[135] Kumar S, Ramkrishna D. On the solution of population balance equations by discretization - I. A fixed pivot technique. Chem. Eng. Sci. 1996;51:1311-1332.

[136] Shu C-W Essentially non-oscillatory and weighted essentially non-oscillatory schemes for hyperbolic conservation laws. NASA/CR-97-206253, ICASE Report No. 97-65. Hampton. 1997.

[137] Kurganov A, Noelle S, Petrova G. Semidiscrete central-upwind schemes for hyperbolic conservation laws and Hamilton-Jacobi equations. SIAM J. Sci. Comput. 2001;23:707-740.

[138] Shu C-W High order finite difference and finite volume WENO schemes and discontinuous Galerkin methods for CFD. NASA/CR-2001-210865, ICASE Report No. 2001-11. Hampton. 2001. 
[139] Lim YI, Le Lann JM, Joulia X. Accuracy, temporal performance and stability comparisons of discretization methods for the numerical solution of partial differential equations (PDEs) in the presence of steep moving fronts. Comp. Chem. Eng. 2001;25:1483-1492.

[140] Hounslow MJ, Ryall RL, Marshall VR. A discretized population balance for nucleation, growth, and aggregation. AIChE J. 1988;34:1821-1832.

[141] Vanni M. Approximate population balance equations for aggregation-breakage processes. J. Colloid Interface Sci. 2000;221:143-160.

[142] Kumar S, Ramkrishna D. On the solution of population balance equations by discretization - II. A moving pivot technique. Chem. Eng. Sci. 1996;51:13331342.

[143] Kumar S, Ramkrishna D. On the solution of population balance equations by discretization - III. Nucleation, growth and aggregation of particles. Chem. Eng. Sci. 1997;52:4659-4679.

[144] van Herk AM, German AL. Modeling of emulsion co- and terpolymerizations: Will it ever be possible? Macromol. Theory Simul. 1998;7:557-565.

[145] Fortuny-Heredia M. Modélisation de procédés pour la synthèse de latex multipopulés. Lyon:Université Claude Bernard Lyon I, 2002.

[146] Herrera-Ordóñez J, Olayo R. On the kinetics of styrene emulsion polymerization above CMC. A mathematical model. J. Polym. Sci., Part A: Polym. Chem. 2000;38:2201-2218.

[147] Sood A. Particle size distribution control in emulsion polymerization. J. Appl. Polym. Sci. 2004;92:2884-2902. 


\section{Figures}

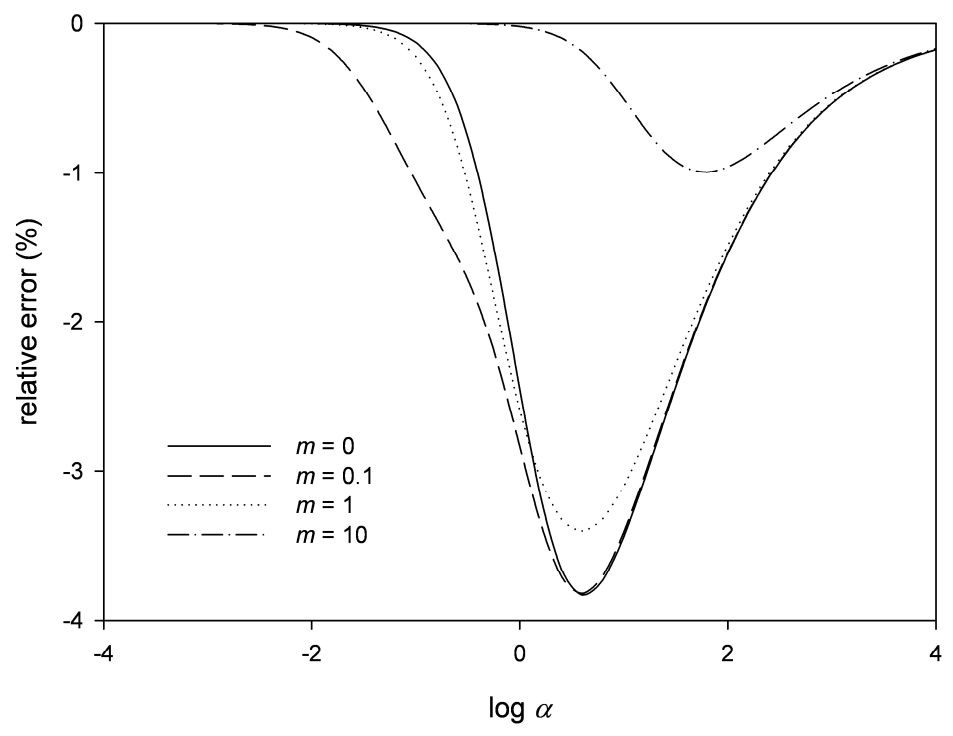

Fig. 1 Relative error of the $\bar{n}$ values computed by Eq. (22) with respect to Eq. (18).

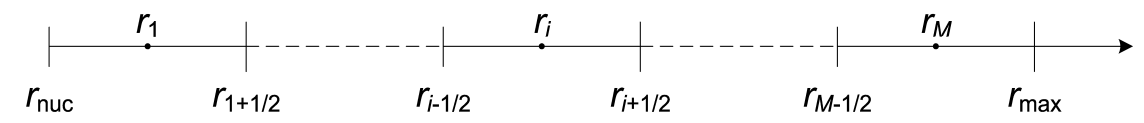

Fig. 2 Subdivision of the particle size domain $\Omega_{r} \equiv\left[r_{n u c}, r_{\max }\right]$ into $M$ cells. 


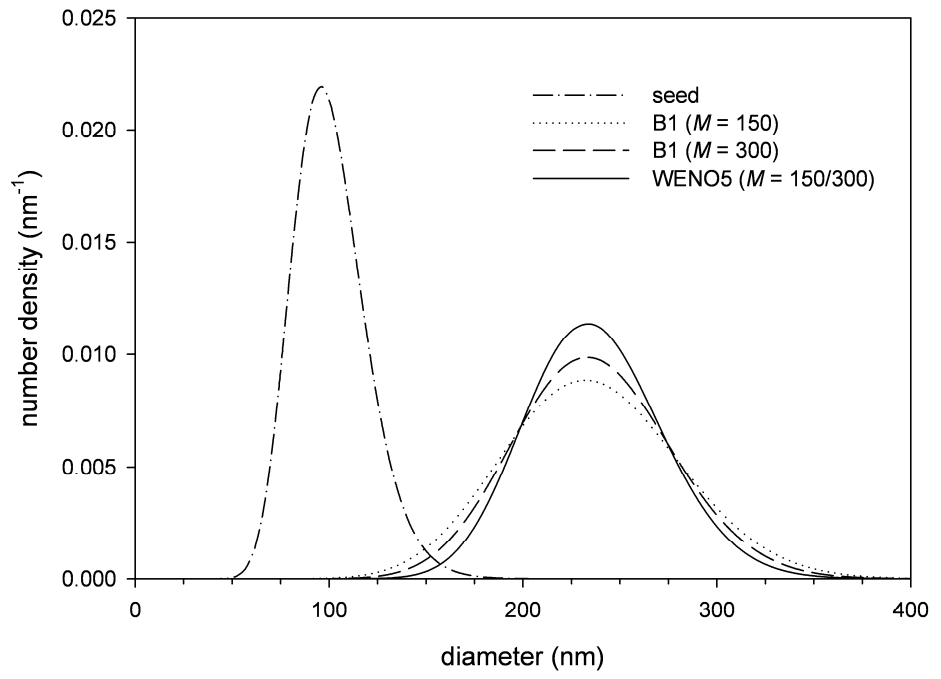

Fig. 3. Growth of a $100 \mathrm{~nm}$ seed. Numerical results obtained with the fifth order WENO and first order upwind schemes, for two values of $M$ (uniform grids).

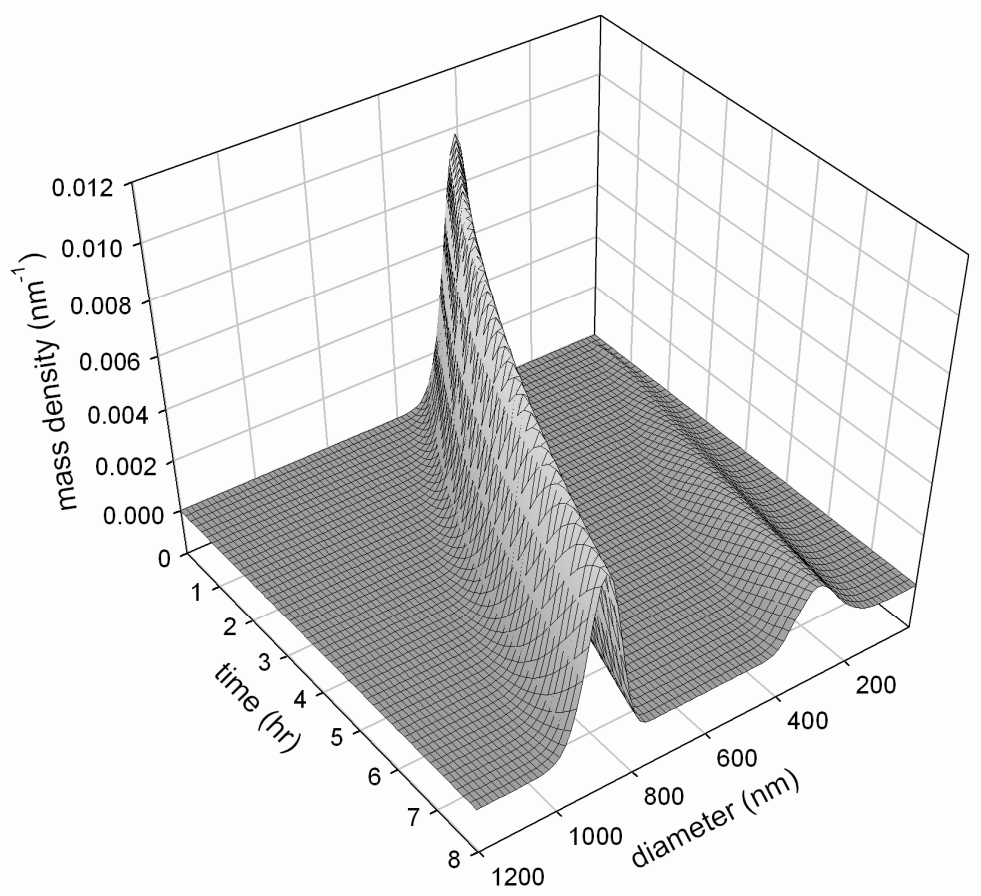

Fig. 4. Synthesis of a bimodal latex by seeded polymerization. Evolution of the massaveraged PSD along the course of the batch. 


\section{Tables}

Table 1 Population balance models of emulsion polymerization

\begin{tabular}{|c|c|c|c|c|c|c|}
\hline Authors & Reactor & System & Kinetics & Coagulation & $\begin{array}{c}\text { Experimental } \\
\text { validation }\end{array}$ & $\begin{array}{c}\mathrm{PBE} \\
\text { solution }\end{array}$ \\
\hline Abad et al. (1994) [44] & loop & VAc/Veova 10 & PB & $\mathrm{N}$ & $\mathrm{Y}$ & OCFE \\
\hline Abad et al. (1995) [45] & loop & VAc/Veova 10 & PB & $\mathrm{N}$ & Y & OCFE \\
\hline Araújo et al. (2001) [12] & loop & VAc/Veova 10 & PB & $\mathrm{Y}$ & Y & OCFE \\
\hline Coen et al. (1998) [8] & batch & Sty & $0-1$ & $\mathrm{Y}$ & $\mathrm{Y}$ & $\mathrm{FD}-\mathrm{C} 2$ \\
\hline Coen et al. (2004) [22] & batch & $\mathrm{BuA}$ & $0-1 / \mathrm{PB}$ & $\mathrm{Y}$ & Y & FD-C2 \\
\hline Crowley et al. (2000) [30] & semibatch & Sty & $0-1$ & $\mathrm{~N}$ & $\mathrm{~N}$ & OCFE \\
\hline Forcolin et al. (1999) [77] & batch & VCM & PB & Y & Y & FD-B1 \\
\hline Fortuny-Heredia (2002) [145] & semibatch & MMA/BuA & $\mathrm{PB}$ & Y & Y & FD-B1 \\
\hline Herrera-Ordóñez and Olayo (2000) [146] & batch & Sty & $\mathrm{PB}$ & $\mathrm{N}$ & Y & FV-B1 \\
\hline Immanuel at al. (2002) [47] & semibatch & $\mathrm{VAc} / \mathrm{BuA}$ & PB & $\mathrm{N}$ & Y & OCFE \\
\hline Immanuel et al. (2003) [13] & semibatch & $\mathrm{VAc} / \mathrm{BuA}$ & $\mathrm{PB}$ & Y & Y & FV-B1 \\
\hline Kammona et al. (2003) [48] & batch & Sty/2-EHA & $\mathrm{PB}$ & $\mathrm{Y}$ & Y & DPB \\
\hline Kiparissides et al. (2002) [86] & batch & VCM & $\mathrm{PB}$ & Y & Y & FD-B1 \\
\hline Meadows et al. (2003) [31] & semibatch & Sty & $0-1$ & $\mathrm{~N}$ & $\mathrm{~N}$ & OCFE \\
\hline Melis et al. (1998) [87] & batch & VCM & $\mathrm{PB}$ & $\mathrm{Y}$ & $\mathrm{N}$ & FD-B1 \\
\hline Saldívar and Ray (1997) [46] & batch/CSTR & Sty/MMA & PB & $\mathrm{N}$ & Y & OCFE \\
\hline Saldívar et al. (1998) [4] & semibatch/CSTR & various & $\mathrm{PB}$ & $\mathrm{N}$ & Y & OCFE \\
\hline Sood (2004) [147] & semibatch & Sty & PB & $\mathrm{N}$ & $\mathrm{N}$ & OCFE \\
\hline Unzueta and Forcada (1997) [121] & semibatch & MMA/BuA & PB & $\mathrm{Y}$ & Y & MM \\
\hline Zeaiter et al. (2002) [29] & semibatch & Sty & $0-1$ & Y & Y & FD-B1 \\
\hline
\end{tabular}

